\title{
A mixed Euler-Euler/Euler-Lagrange approach to erosion prediction
}

\author{
Gianandrea Vittorio Messa*, Giacomo Ferrarese, Stefano Malavasi
}

Dipt. I.C.A., Politecnico di Milano, Piazza Leonardo da Vinci, 32, 20133 Milano, Italy

\begin{abstract}
Providing quantitative assessment of erosion in the various parts of a piping system is of considerable importance in the oil\&gas industry. Field and lab erosion testing are extremely onerous and, for this reason, this task is typically achieved by means of algebraic erosion correlations in conjunction with CFD two-phase models based on the Euler-Lagrange approach for flow computation. However, the high computational burden makes this approach onerous even for relatively simple benchmark cases, and it cannot be actually applied in many practical applications. In this paper, we present an innovative approach to erosion prediction, which relies on the combined use of Euler-Euler and Euler-Lagrange CFD two-phase models. The strength of the proposed approach, compared to the standard practice, resides in its numerical efficiency, arising from the fact that the Lagrangian description of the solid phase is restricted to certain subdomains bounded by the surfaces most vulnerable to erosion. The outcomes of two application cases, namely an abrasive jet impingement test and a needle and seat choke valve, demonstrate that the new approach allows considerable reduction of the computational burden for particle tracking and wear estimation. This will open the way for addressing more complex flows of considerable interest in practical applications, which are actually precluded at present.
\end{abstract}

Keywords: Erosion prediction, Euler-Euler models, Euler-Lagrange models, abrasive jets, choke valves.

\footnotetext{
${ }^{*}$ Corresponding author.

Dept. ICA - Politecnico di Milano

Piazza Leonardo da Vinci, 32

20133 Milano (Italy)

Tel: +3902 23996287

E mail-address: gianandreavittorio.messa@ polimi.it
} 


\section{Introduction}

The erosion caused by the impacts of solid particles dragged by a liquid is a serious concern in many engineering applications. A significant example is given by the oil plants, where the erosion of the wellhead chokes valves and of the pipeline components is likely to occur due to the presence of solid particles within the flow. The relevance of erosion in the oil\&gas industry is made evident in a paper by Wood et al. [1] who, making reference to an earlier report by British Petroleum, wrote that costs can be as high as $£ 500,000$ for subsea replacements and that in extreme cases pipeline components are eroded away in months. Particularly, the most important erosion-related problems are the identification of the erosion hotspot locations and the estimation of the loss of material under aggressive flow conditions.

Performing field and lab erosion testing for providing the erosion characterization of pipeline components is extremely hard due to economic and technical difficulties. The few researches reported in the literature mainly refer to benchmark cases like abrasive jet impingement tests [2-9] or simple pipe fittings [4,10-12]. Experimental studies concerning complex devices such as choke valves are even rarer [13-17]. For this reason, disposing of tools for erosion prediction is of considerable interest for engineers in the design stage as well as in the management one.

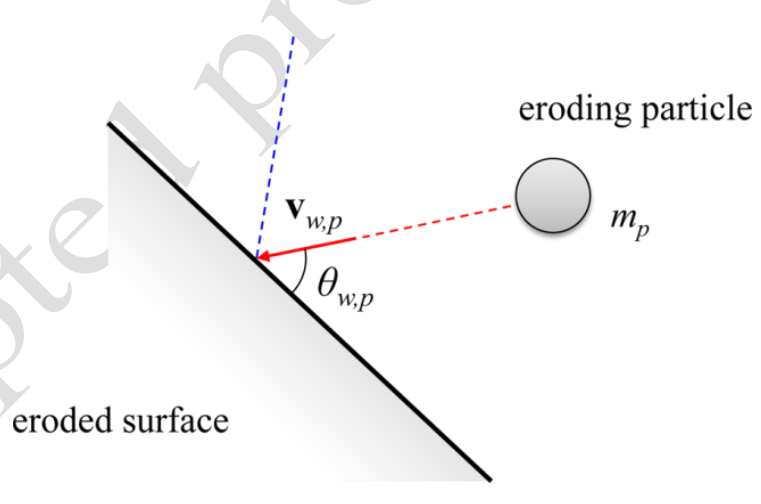

Fig. 1 An eroding particle hitting a surface.

At present, the standard practice involves the use of "single-particle" erosion correlations, which treat the erosion process in terms of the interaction between each eroding particle and a surface, and express the mass of eroded material removed $E_{p}$ as a function of the particle mass $m_{p}$, the modulus of the particle impact speed vector $\left|\mathbf{v}_{p, w}\right|$, the particle impact angle $\theta_{w, p}$, and some properties of the materials involved in the erosion process (Fig. 1). Typically the erosion correlations are of empirical nature and they are obtained by fitting the outcomes 
of laboratory tests in which an abrasive jet impinges at high velocity against a specimen of the target material, even if this practice is still one of the most restricting uncertainties in erosion prediction [15]. The reviews of the available "single-particle" erosion correlations performed by Lyczkowski and Bouillard [18] and Parsi et al. [19] evidence the similar mathematical structure of these models, which mainly differ from the values of their empirical constants. In this work we will employ three erosion correlations, namely the E/CRC equation [5], the DNV correlation [20], and the equation of Oka et al. [21,22]. These correlations will be illustrated in Subsection 2.3.

The fluid dynamic properties of the particles at the impact stage, $\left|\mathbf{v}_{p, w}\right|$ and $\theta_{w, p}$, are usually obtained from CFD simulations based on the Euler-Lagrange (EL) approach under the assumption of "one-way coupling" regime, in which the fluid flow without solids is first simulated in an Eulerian framework (i.e. cell-based), and, afterwards, the solid phase is represented in a Lagrangian framework by tracking the trajectories of a certain number of parcels (i.e. group of particles sharing the same fluid-dynamic properties). This approach provides rather accurate estimates of the two-phase flow field, but it is extremely onerous from a computational point of view. In fact, since the computed trajectories are random due to the dispersive action of the fluid turbulence, many parcels have to be tracked in order to have significant wear statistics. In previous erosion analyses on different geometries [12,15,23-25], the number of injected parcels is always of the order of tens of thousands. This leads to a huge amount of calculated data, especially in case of complex devices where the tracking of the trajectories is very resource consuming, even if only the particle properties close to the walls are actually needed for erosion prediction purposes. The already high computational burden becomes prohibitive when some restrictive hypotheses on the flow and erosion processes are not met, to the point of precluding the practical possibility of performing erosion predictions. This typically happens in two situations. First, when the mass loading, i.e. the particle mass per unit volume of mixture divided by the continuous-fluid mass per unit volume of mixture, exceeds about $10^{-3}$ [26], because the particles start influencing the flow of the fluid ("two-way coupling" regime) and, to an even greater extent, for particle volume fractions above $10^{-2}$ [27], because the interactions among the particles have to be accounted for as well ("four-way coupling" regime). As far as we know, no attempt of predicting the erosion of a valve caused by dense mixtures has been reported in the literature except for a relatively recent study in which the geometry of the device had been extremely simplified and modeled as two-dimensional [28]. Second, when the geometry changes due to erosion significantly affects the evolution of the erosion process, as it typically happens when a device is subjected to aggressive flow conditions for a prolonged period. This prevents the two phase flow to be simulated as steady-state. A CFD model 
accounting for geometry changes dynamically does not seem available at present, the study by Nguyen et al. [6] being one of the firsts addressing this aspect. The literature available indicates that, for a large set of geometries, the above reported approach is capable in reproducing the erosion hotspot locations but fails in predicting correctly the amount of removed material $[6,12,15,17]$, and this has been mainly attributed to the employment of inadequate erosion correlations as well as the neglect of the geometry changes due to erosion.

In this work we present an alternative approach which combines the Lagrangian tracking of the particles in the proximity of the erosion hotspots locations with an Euler-Euler two-phase CFD model which solves only for the average properties of the ensemble of particles. CFD models of this type are usually employed for simulating dense flows $[29,30]$, and they have been successfully applied to mixtures with lower solid loading as well [31]. However, EE models are not currently used for erosion prediction in oil\&gas applications, because the erosion correlations require as input the characteristics of each eroding particle, which these CFD models don't provide. In order to validate the new approach, the wear estimates are compared with those obtained with the standard CFD-based erosion prediction practice (in which the parcels' trajectories are determined in the whole computational domain) which, due to its considerable computational burden, is actually dependent upon the fulfilment of the already mentioned restrictive assumptions, namely low solid loading and negligible geometry changes due to erosion, especially when applied to complex geometries [14,15,17,32].

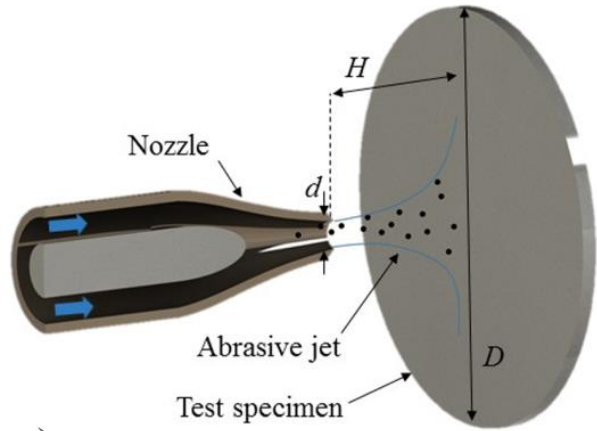

a)

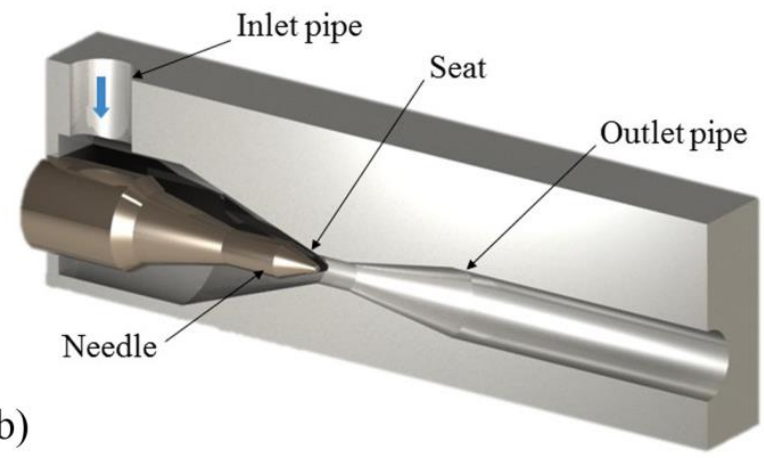

Fig. 2 The two test cases considered in this study: a) abrasive jet impingement test; b) needle and seat valve.

Two test cases are considered. The former consists of abrasive jet impingement test (Fig. 2a) and, particularly, we reproduced the experiments carried out by Okita et al. [5] and employed their data for validation purposes. The latter is a simplified model of a needle and seat choke valve (Fig $2 b$ ). 
The remainder of this paper is divided in three sections, followed by the conclusions. The mathematical models are first described, together with the numerical setup and the computational methodology. The mixed Euler-Euler / Euler-Lagrange approach is then illustrated and, afterwards, applied to the two test cases reported above.

\section{Mathematical models}

In this work we made use of the Euler-Lagrange and the Euler-Euler CFD models embedded in the PHOENICS commercial code version 2011. However, it is worth noticing that the used models, which are illustrated in the following, are the typical ones that are available in the other general-purpose CFD codes; therefore, we would expect similar results to be produced by other codes. We also underline that the proposed methodology is applicable regardless of the specific characteristics of the employed two-phase CFD models as well as the used erosion correlation.

\subsection{Euler-Lagrange modelling}

As already noticed, the low mass loadings considered in this study enabled the possibility of running the EL simulations under the hypothesis of "one-way coupling" regime, in which the fluid affects the motion of the particles but not vice-versa. Therefore, the fluid flow field is obtained from single-phase flow computations by solving the well-known RANS equations [33] coupled with the standard high Reynolds form of the $k-\varepsilon$ turbulence model of Launder and Spalding [34] to evaluate the Reynolds stress tensor. Solution of these equations yields the mean velocity vector of the fluid $\mathbf{U}_{E L}$, the mean pressure of the fluid $P_{E L}$, the turbulent kinetic energy $k_{E L}$, and its dissipation rate $\varepsilon_{E L}$.

Under the hypothesis that the parcels do not interact with each other, the Lagrangian tracking calculations are performed by solving the following set of ordinary differential equation for each parcel:

$$
\left\{\begin{array}{l}
\frac{d \mathbf{x}_{E L}}{d t}=\mathbf{v}_{E L} \\
\frac{d \mathbf{v}_{E L}}{d t}=\frac{3}{4 d_{p}} \frac{\rho_{f}}{\rho_{p}} C_{d}\left|\tilde{\mathbf{u}}_{E L}-\mathbf{v}_{E L}\right|\left(\tilde{\mathbf{u}}_{E L}-\mathbf{v}_{E L}\right)+\frac{1}{\rho_{p}} \tilde{\nabla} P_{E L}
\end{array}\right.
$$

where $\mathbf{x}_{E L}$ is the parcel position vector, $\mathbf{v}_{E L}$ is the instantaneous parcel velocity vector, $d_{p}$ is the particle diameter, $C_{d}$ is the drag coefficient, $\rho_{f}$ and $\rho_{p}$ are the densities of fluid and particles respectively, and the tilde symbol indicates that the instantaneous fluid velocity $\mathbf{u}^{E L}$ and the gradient of the mean fluid pressure $\nabla P_{E L}$ are evaluated 
at parcel position. This is obtained by interpolation of the known values in the nearest nodes. The drag coefficient is given by the well-known correlation of Shiller and Naumann [35]:

$$
C_{d}=\max \left\{\frac{24}{R e_{p, E L}}\left[1+0.15\left(R e_{p, E L}\right)^{0.687}\right], 0.44\right\}
$$

in which $\operatorname{Re}_{p, E L}=\rho_{f} d_{p}\left|\tilde{\mathbf{u}}_{E L}-\mathbf{v}_{E L}\right| / \mu$ is the particle Reynolds number, and $\mu$ is the viscosity of the fluid. Other forces, such as gravity, Saffman lift, Magnus lift, virtual mass, and history force, were neglected being apparently related to side effects of minor importance for the case study under consideration. In order to account for the turbulent dispersion of the particles, the stochastic turbulence model of Gosman and Ioannides [36] is employed for the evaluation of $\mathbf{u}_{E L}$, which is expressed as the sum of the fluid average velocity $\mathbf{U}_{E L}$ plus a fluctuation $\mathbf{u}_{E L}$ calculated assuming that its components follow a Gaussian distribution with zero mean value and a standard deviation equal to $\left(2 k_{E L} / 3\right)^{1 / 2}$. The fluctuating component $\mathbf{u}_{E L}^{\prime}$ is assumed to act over a time interval $\Delta t_{\text {int }}$ which is the minimum of the eddy life-time $\Delta t_{e}$ and the transit time $\Delta t_{r}$ taken by a parcel to cross the eddy. $\Delta t_{e}$ and $\Delta t_{r}$ are evaluated as follows:

$$
\Delta t_{e}=\frac{l_{e}}{\left|\tilde{\mathbf{u}}_{E L}\right|} \quad \Delta t_{r}=\frac{l_{e}}{\left|\tilde{\mathbf{u}}_{E L}-\mathbf{v}_{E L}\right|} \quad l_{e}=C_{\mu}^{3 / 4} \frac{\left(\tilde{k}_{E L}\right)^{3 / 2}}{\tilde{\varepsilon}_{E L}}
$$

where $l_{e}$ is the mixing length, and $C_{\mu}$ is one of the numerical constants of the standard $k$ - $\varepsilon$ turbulence model for high Reynolds number flows [34].

\subsection{Euler-Euler modelling}

In the EE approach both phases are interpreted as interpenetrating continua and the average fluid dynamic properties of the ensemble of particles, referred to as "solid phase", are solved in an Eulerian framework. The conservation equations of the two phases are solved coupled but, due to the low particle loading of the test cases of the present study, the fluid flow field computed by the EE model is actually indistinguishable for that of a single-phase flow simulation. The EE model used in this work was developed by Spalding [37] and embedded in the PHOENICS code. This model solves the following set of steady-state mass and momentum conservation equations for the fluid and the solid phases, respectively:

$$
\begin{gathered}
\nabla \cdot\left[\rho_{f}\left(1-\Phi_{E E}\right) \mathbf{U}_{E E}\right]-\nabla \cdot\left(\rho_{f} \overline{\phi_{E E}^{\prime} \mathbf{u}_{E E}^{\prime}}\right)=0 \\
\nabla \cdot\left(\Phi_{E E} \rho_{p} \mathbf{V}_{E E}\right)+\nabla \cdot\left(\rho_{p} \overline{\phi_{E E}^{\prime} \mathbf{v}_{E E}^{\prime}}\right)=0
\end{gathered}
$$




$$
\begin{aligned}
& \nabla \cdot\left[\rho_{f}\left(1-\Phi_{E E}\right) \mathbf{U}_{E E} \mathbf{U}_{E E}\right]-\nabla \cdot\left[\left(1-\Phi_{E E}\right)\left(\mu+\mu_{E E}^{t}\right) \nabla \mathbf{U}_{E E}\right]= \\
&+\nabla \cdot\left(\rho_{f} \overline{\phi_{E E}^{\prime} \mathbf{u}_{E E}^{\prime}} \mathbf{U}_{E E}\right)=-\left(1-\Phi_{E E}\right) \nabla P_{E E}+\mathbf{M}_{E E} \\
& \nabla \cdot\left(\rho_{p} \Phi_{E E} \mathbf{V}_{E E} \mathbf{V}_{E E}\right)-\nabla \cdot\left(\Phi_{E E} \mu_{s, E E}^{t} \nabla \mathbf{V}_{E E}\right)-\nabla \cdot\left(\rho_{p} \overline{\phi_{E E}^{\prime} \mathbf{v}_{E E}^{\prime}} \mathbf{V}_{E E}\right)=-\Phi_{E E} \nabla P_{E E}-\mathbf{M}_{E E}
\end{aligned}
$$

where: $\Phi_{E E}$ and $\phi^{\prime}{ }_{E E}$ are the mean and fluctuating solid volume fraction; $\mathbf{U}_{E E}$ and $\mathbf{u}_{E E}$ are the mean and fluctuating fluid velocity vectors; $\mathbf{V}_{E E}$ and $\mathbf{v}_{E E}$ are the mean and fluctuating velocity vectors of the solid phase; $P_{E E}$ is the mean pressure, shared by the phases; $\mu_{E E}^{t}$ and $\mu_{s, E E}^{t}$ is the eddy viscosities of the fluid and solid phases, respectively; and $\mathbf{M}_{E E}$ is the generalized drag term, which accounts for the momentum transfer between the phases. For consistency with the EL simulations, $\mathbf{M}_{E E}$ includes only the drag force and it is therefore given by:

$$
\mathbf{M}_{E E}=\frac{3}{4 d_{p}} \rho_{f} \Phi_{E E} C_{d}\left|\mathbf{U}_{E E}-\mathbf{V}_{E E}\right|\left(\mathbf{U}_{E E}-\mathbf{V}_{E E}\right)
$$

with the drag coefficient evaluated by means of the correlation of Shiller and Neumann [35], thereby applying Eq. (2) after replacing $R e_{p, E L}$ with $R e_{p, E E}=\rho_{f} d_{p}\left|\mathbf{U}_{E E}-\mathbf{V}_{E E}\right| / \mu$.

The correlations between the fluctuating velocities and the fluctuating volume fractions are modeled by means of the eddy diffusivity hypothesis, as follows:

$$
\overline{\phi_{E E}^{\prime} \mathbf{u}_{E E}^{\prime}}=\overline{\phi^{\prime}{ }_{E E} \mathbf{v}^{\prime}{ }_{E E}}=\frac{\mu_{E E}^{t}}{\rho_{f} \sigma_{\Phi}} \nabla \Phi_{E E}
$$

where $\sigma_{\Phi}$ is the turbulent Schmidt number for volume fractions, which is assigned a unit value in this study. The eddy viscosity $\mu_{E E}^{t}$ is evaluated by means of the following extension [38,39] of the standard high Reynolds form of the $k-\varepsilon$ turbulence model of Launder and Spalding [34] to two-phase flow:

$$
\begin{gathered}
\nabla \cdot\left[\rho_{f}\left(1-\Phi_{E E}\right) k_{E E} \mathbf{U}_{E E}\right]-\nabla \cdot\left(\rho_{f} \overline{\phi^{\prime}{ }_{E E} \mathbf{u}_{E E}^{\prime}} k_{E E}\right)= \\
\nabla \cdot\left[\left(1-\Phi_{E E}\right)\left(\mu+\frac{\mu_{E E}^{t}}{\sigma_{k}}\right) \nabla k_{E E}\right]+\left(1-\Phi_{E E}\right) \rho_{f}\left(P_{k, E E}-\varepsilon_{E E}\right) \\
\nabla \cdot\left[\rho_{f}\left(1-\Phi_{E E}\right) \varepsilon_{E E} \mathbf{U}_{E E}\right]-\nabla \cdot\left(\rho_{f} \overline{\phi^{\prime}{ }_{E E} \mathbf{u}_{E E}^{\prime}} \varepsilon_{E E}\right)= \\
\nabla \cdot\left[\left(1-\Phi_{E E}\right)\left(\mu+\frac{\mu_{E E}^{t}}{\sigma_{\varepsilon}}\right) \nabla \varepsilon_{E E}\right]+\left(1-\Phi_{E E}\right) \rho_{f} \frac{\varepsilon_{E E}}{k_{E E}}\left(C_{1 \varepsilon} P_{k, E E}-C_{2 \varepsilon} \varepsilon_{E E}\right) \\
\mu_{E E}^{t}=\rho_{f} C_{\mu} \frac{k_{E E}^{2}}{\varepsilon_{E E}}
\end{gathered}
$$

in which $P_{k, E E}=\mu_{E E}^{t} / \rho_{f} \cdot\left[\nabla \mathbf{U}_{E E}+\left(\nabla \mathbf{U}_{E E}\right)^{+}\right]: \nabla \mathbf{U}_{E E}$ is the volumetric production rate of $k$ due to the working of the Reynolds stresses against the mean flow (the superscript "+" indicates that the transpose of the dyadic $\nabla \mathbf{U}_{E E}$ is taken), and the numerical constants $\sigma_{k}, \sigma_{\varepsilon}, C_{l \varepsilon}$, and $C_{2 \varepsilon}$ are given the standard values [34], namely 1.0. 
$1.314,1.44$, and 1.92 respectively. The algebraic particle turbulence model of Hinze [40] was employed to relate the eddy viscosity of the solid phase $\mu_{s, E E}^{t}$ to that of the fluid $\mu_{E E}^{t}$, as follows:

$$
\mu_{s, E E}^{t}=\frac{\rho_{p}}{\rho_{f}} \frac{\mu_{E E}^{t}}{1+S t}
$$

where $S t$ is the particle Stokes number, which is defined as the ratio between the response time of a particle $\tau_{p}$ and a characteristic fluid time scale $\tau_{f}$. Here $S t$ is given a constant value. The response time of a particle $\tau_{p}$ is calculated as:

$$
\tau_{p}=\frac{\left(\rho_{p}+C_{v m} \rho_{f}\right) d_{p}^{2}}{18 \mu\left(1+0.15 \overline{R e}_{p}^{0.687}\right)}
$$

where $C_{v m}=0.5$ is the virtual mass coefficient, and the particle Reynolds number $\overline{\operatorname{Re}}_{p}$ is the one characteristic of a single particle falling in a quiescent liquid. The fluid time scale $\tau_{f}$ is defined in terms of integral properties, and will be from time to time specified for the two case studies.

\subsection{Erosion correlations}

As already mentioned, in this study we will make use of three different erosion correlations, as follows. The correlation developed at the Erosion/Corrosion Research Center of the University of Tulsa, referred to as E/CRC equation, has been employed in the abrasive jet case, since it has been calibrated by Okita et al. [5] for the specific flow condition object of investigation. This correlation expresses the mass of eroded material removed by an impinging particle as:

$$
E_{p}=m_{p} F_{s} C(B H)^{-0.59}\left|\mathbf{v}_{p, w}\right|^{n} F\left(\theta_{w, p}\right)
$$

where: $F_{s}$ is a sharpness factor depending on the shape of the eroding particles; $C$ and $n$ are empirical constants; and $\mathrm{BH}$ is the Brinell hardness of the target material calculated based on the Vicker's hardness $H_{v}$ as:

$$
B H=\frac{H_{v}+0.1023}{0.0108}
$$

The angle function $F\left(\theta_{w, p}\right)$ is given by the following equation:

$$
F\left(\theta_{w, p}\right)=\frac{1}{f} \cdot\left(\sin \theta_{w, p}\right)^{n 1}\left[1+H_{v}^{n 3}\left(1-\sin \theta_{w, p}\right)\right]^{n 2}
$$

where $n 1, n 2$, and $n 3$ are empirical constants and $f$ is the maximum value of $F\left(\theta_{w, p}\right)$ used to normalize the function. 
The DNV [20] erosion correlation has been selected for use in the needle and seat valve case, being of considerable practical relevance in the oil\&gas sector. The equation is as follows:

$$
E_{p}=m_{p} K\left|\mathbf{v}_{p, w}\right|^{n} F\left(\theta_{w, p}\right)
$$

where $K$ and $n$ are constants depending on the material and the angle function $F\left(\theta_{w, p}\right)$ is given by:

$$
\begin{gathered}
F\left(\theta_{w, p}\right)=9.370 \cdot \theta_{w, p}-42.295 \cdot \theta_{w, p}^{2}+110.864 \cdot \theta_{w, p}^{3}-175.804 \cdot \theta_{w, p}^{4}+ \\
+170.137 \cdot \theta_{w, p}^{5}-98.398 \cdot \theta_{w, p}^{6}+31.211 \cdot \theta_{w, p}^{7}-4.170 \cdot \theta_{w, p}^{8}
\end{gathered}
$$

In order to analyze the influence of the used erosion correlation, the wear estimates in the valve case have been repeated by employing the formula developed by Oka et al. [21,22], which is one of the most commonly used in recent erosion studies [4-6,24]. Oka's correlation says that:

$$
E_{p}=10^{-9} m_{p} \rho_{w} K\left(H_{v}\right)^{k 1}\left(\frac{\left|\mathbf{v}_{p, w}\right|}{V^{\prime}}\right)^{k 2}\left(\frac{d_{p}}{D^{\prime}}\right)^{k 3} F\left(\theta_{w, p}\right)
$$

where $\rho_{w}$ is the density of the target material, $V^{\prime}$ is the particle reference velocity, $d_{p}$ and $D^{\prime}$ are the particle diameter and reference diameter, respectively, and $k 1, k 2=2.3\left(H_{v}\right)^{0.038}, k 3$ are the exponent factors, and $K$ is the particle property factor. The angle function $F\left(\theta_{w, p}\right)$ is given by the following equation:

$$
F\left(\theta_{w, p}\right)=\left(\sin \theta_{w, p}\right)^{n 1}\left[1+H_{v}\left(1-\sin \theta_{w, p}\right)^{n 2}\right]
$$

where $n 1=s 1\left(H_{v}\right)^{q 1}, n 2=s 2\left(H_{v}\right)^{q 2}$, and $n 3$ are exponents determined by the material hardness and other impact conditions, and $s 1, s 2, q 1, q 2$ are the fitting constants for the particle material.

\subsection{Computational domain and boundary conditions}

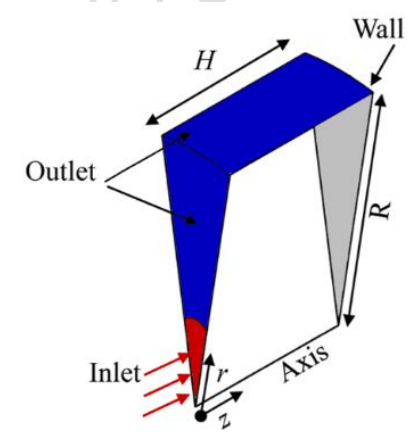

(a)

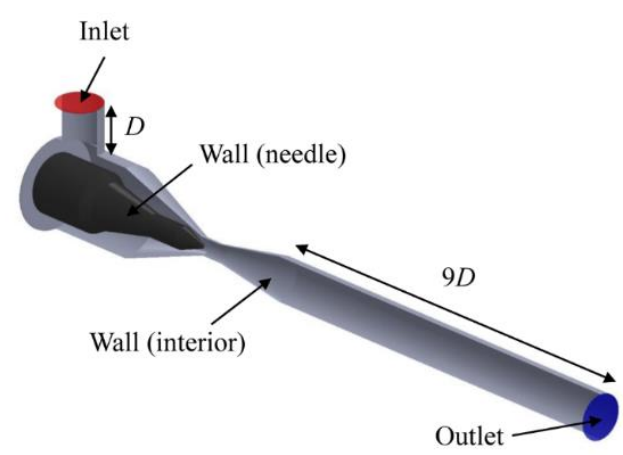

(b)

Fig. 3 Computational domain and boundary conditions for the two test cases: (a) abrasive jet impingement test; (b) needle and seat valve. 
The computational domains for the abrasive jet impingement test and the needle and seat valve are reported in Figs. 3(a) and (b) respectively. In abrasive jet case, the flow and geometrical symmetries of the phenomenon have been exploited by solving only over a thin slice of the jet with one cell in the azimuthal direction. Strictly speaking, the particle tracking in the EL computations should be always performed in a 3D domain due to the $3 \mathrm{D}$ effect of turbulence, but nevertheless in many studies the symmetries of the mean flow are improperly exploited in order to reduce the computational burden of the simulations $[4,5]$. We run 3D simulations too, and verified the good correspondence between the two solutions. In the complex geometry case, the geometrical symmetry of the valve with respect to the mid plane could not be exploited because of the strong swirl occurring in the valve body and the downstream pipe, a very critical feature in valve flow simulations [41].

The boundary conditions applied in both the EL and EE simulations are now discussed, in the following order: inlet boundaries, solid walls, outlet boundaries, and axes of symmetry.

\section{1) Inlet boundaries}

The conditions applied to the fluid variables at the inlet section are different in the two simulations scenarios. In fact, in the abrasive jet case a fully developed turbulent flow profile is specified at the pipe inlet, with the distribution of the mean axial fluid velocity, turbulent kinetic energy, and dissipation rate determined from Nikuradse's boundary-layer theory [42] for single-phase flow in straight pipes. Conversely, in the valve case the fluid axial velocity was uniformly distributed, whilst the turbulent kinetic energy and its dissipation rate were derived from a turbulent intensity of $5 \%$ and an inlet mixing length equal to $7 \%$ of the pipe diameter. It is worth noticing that the short inlet pipe length simulated $(1 D$, as shown in Fig. 3(b)) does not allow attaining fully-developed flow conditions at the valve entrance, but this is unlikely to have any effect on the two-phase flow field within the valve body, thereby affecting the quality of our erosion estimates. For both the simple geometry and the complex one, no mean relative velocity occurs between the phases at the inlet boundary. In the EE simulations the same mean velocity is applied to the fluid and the particles (i.e. $\mathbf{V}_{E E}=\mathbf{U}_{E E}$ at the inlet boundary), and the mean solid volume fraction is taken as uniformly distributed. In the EL simulations, the initial parcel velocity $\mathbf{v}_{E L, 0}$ was equal to the mean fluid velocity plus a fluctuation, as follows:

$$
\mathbf{v}_{E L, 0}=\tilde{\mathbf{U}}_{E L}+\xi\left(\frac{2 \tilde{k}_{E L}}{3}\right)^{1 / 2}
$$


where $\xi$ is a three-element vector containing random scalars drawn from the standard PDF. The initial parcels' positions were determined for the local parcel area number density to be proportional to the advective fluid mass flux per unit area, i.e. $\rho_{f} \mathbf{U}_{E L} \cdot \mathbf{n}$ (n is the unit inward normal vector to the inlet boundary). All parcels are attributed the same mass flux, which is determined in such a way that the total solid mass flux equals the desired value.

2) Outlet boundaries

At the outlet boundary the mean pressure is specified and held constant to a zero value. In the EL simulations, the parcels simply leave the domain, whilst in the EE ones the solid volume fraction and mass outflow of the solid phase result as part of the overall solution. In the valve case, the outlet section is located $9 D$ downstream the diffuser in order to allow a complete pressure recovery (Fig. 3(b)).

3) Solid walls

At the solid walls, no slip is assumed for the fluid phase, and the equilibrium wall function of Launder and Spalding [43] for smooth walls is employed for evaluating the fluid wall shear stress, and the turbulent kinetic energy and its dissipation rate in the near-wall cells. In the EL simulations, the normal and tangential components of the reflected parcel velocity are related to the corresponding incident values via two restitution coefficients. Even if some researches have shown that the restitution coefficients may be angle-dependent [32], we found that simply assigning a unit value to both parameters yields predictions of the particle flow field in agreement with the experimental evidence for the abrasive jet case, which is the only one where laboratory measurements are available.

4) Axes of symmetry

At the axis of symmetry in the abrasive jet case, the normal gradients for all dependent variables are zero, so that a zero-flux condition is applied along such boundary. The treatment of particles is reflection, i.e. bouncing with a restitution coefficient of 1 .

\subsection{Computational methodology and consistency of the numerical solution}

The single-phase RANS and the EE equations have been discretized using the finite volume method, and the calculations performed following the elliptic-staggered formulation. Central differencing is employed for the diffusion terms, while the convection terms are discretized using the hybrid differencing scheme of Spalding

[44]. The finite volume equations are solved iteratively by means of the SIMPLEST [38] and IPSA [37] 
algorithms. The calculation procedure is organized in a slab-by-slab manner, in which all the dependent variables are solved at the current slab before the solver routine moves to the next slab. The numerical solution procedure requires appropriate relaxation of the field variables to achieve convergence. Particularly, inertial relaxation is normally applied to the velocity variables, whereas the latter is applied to all other flow variables, as and when necessary.

Owing to the assumption of "one-way coupling" regime, the parcels' equations are integrating after the solution of the fluid flow field. This is done by means of the GENTRA package version 2010 embedded in the PHOENICS code, supplied by user-made FORTRAN coding allowing the parcels' impact data to be stored. GENTRA sets automatically the Lagrangian time step used to numerically integrate Eq. (1), but the user is allowed to specify a maximum Lagrangian time-step and an approximate number of integration steps for each parcel within each cell. These parameters were chosen as $0.01 \mathrm{~s}$ and 5, respectively, after verifying the negligible dependence of the numerical solution upon the set values. Extensive MATLAB routines have been also developed for the post-processing of the GENTRA output. Particularly, the solid surfaces have been triangulated and each impact point has been associated to the nearest centroid of the surface elements. Application of the erosion correlations (Subsection 2.3) in which the particle mass $m_{p}$ had been replaced by the parcel mass flux $\dot{m}_{P}$ yields the mass flux of eroded material removed by the current parcel. The sum over the parcels yields the erosion rate of each surface element which, once divided by the solid mass flux entering the domain through the inlet section, yields the local erosion ratio $\dot{E}(\mathrm{Kg} / \mathrm{Kg})$. The total erosion ratio $\dot{E}_{\text {tot }}$ can be easily obtained by summing over the surface elements.

The consistency of the numerical solution is now discussed for the two simulated geometries, and the results are shown graphically for the valve case. A cylindrical polar structured mesh with one cell in the azimuthal direction was used to discretize the domain in the abrasive jet case. A grid independence study revealed that a mesh consisting of 211 by 94 cells along the radial and the axial directions, densified close to the solid wall and the symmetry axis, is capable of procuring numerically consistent single-phase and EE solutions. Since the erosion at the walls due to individual parcels' impacts is related to the mass flux $\dot{m}_{P}$ that each particle represents, it is necessary to insure that a statistically representative set of particle impingements is obtained. This is achieved by investigating the sensitivity of the erosion ratio predictions to increasing numbers of tracked parcels. Such an analysis confirmed that, in the abrasive jet impingement test case, 50.000 parcels allowed attaining statistically significant erosion ratio distributions. 


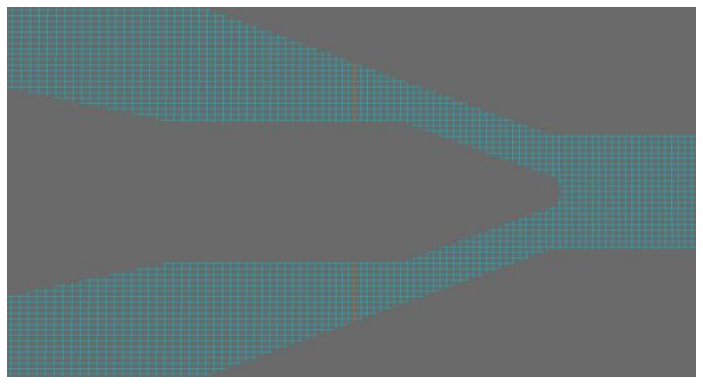

Fig. 4 Detail of the mesh close to the needle tip.

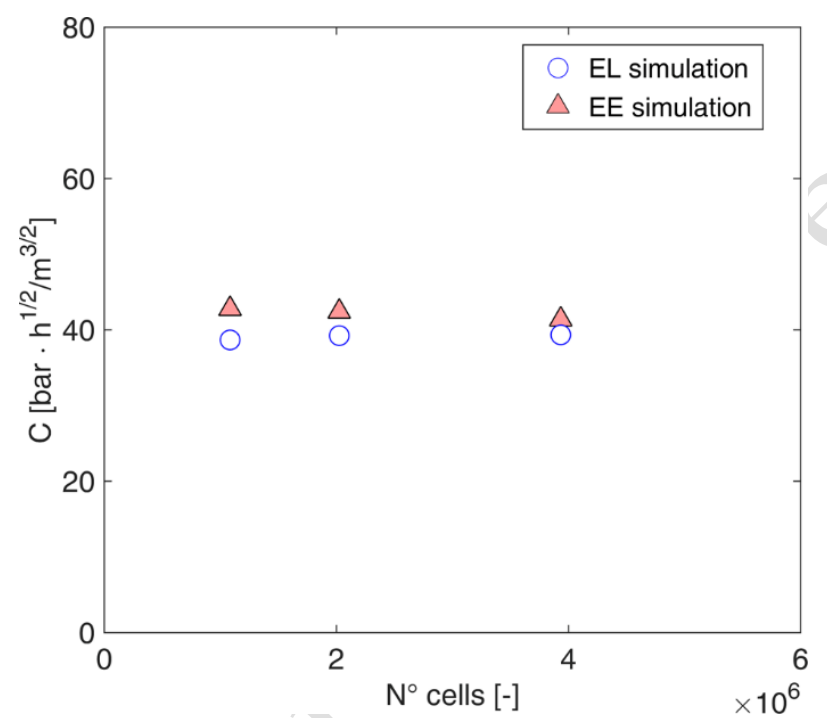

Fig. 5 Effect of the number of cells on the predicted flow coefficient of the needle and seat valve

A structured mesh in Cartesian coordinates is used to discretize the valve domain, and the cut-cell PARSOL technique of PHOENICS was used to treat the volume elements cut by solid surfaces. The mesh consisted of about 4 million cells, the grid being densified within the valve body (Fig. 4). A grid independence analysis was performed to guarantee the consistency of the numerical solution. The flow conditions are those addressed in the subsequent application study (Subsection 4.2), as follows: mixture of water and sand particles with density of $2700 \mathrm{~kg} / \mathrm{m}^{3}$ and size of $400 \mu \mathrm{m}$ at solid volume fraction of $1 \%$; mean flow velocity in the inlet pipe equal to $7 \mathrm{~m} / \mathrm{s}$ (corresponding to a flow rate of $14.2 \mathrm{l} / \mathrm{s}$ ). The flow simulation was repeated employing three different grids in which the number of cells was increased from 1.1 to 4 million, and it was investigated the effect of the number of cells upon the flow coefficient $C$ introduced in the IEC standard [45] to quantify the regulation characteristics of the device: 


$$
C=Q \sqrt{\frac{\rho_{f} / \rho_{f, 0}}{\Delta P}}
$$

where $Q$ is the volume flow rate, $\rho_{f, 0}$ is the fluid density at a temperature of $15^{\circ} \mathrm{C}$, and $\Delta P$ is the pressure drop, evaluated as the difference between the mean pressure at the inlet section and that at a distance of $6 D$ downstream the diffuser. The choice of considering the flow coefficient as the target parameter for the grid independence study is a consequence of the significance of this variable which, as reported in previous studies [15], indicates primarily the pressure losses through the valve, but it is also straightly connected to the acceleration effects that influence the erosion conditions directly. The results, shown in Fig. 5, reveal that as the number of cells increases the predicted $C$ approaches a constant value, which is almost the same for both the EL and EE simulations. Therefore, the used mesh proved capable in providing consistent estimation of the flow coefficient.

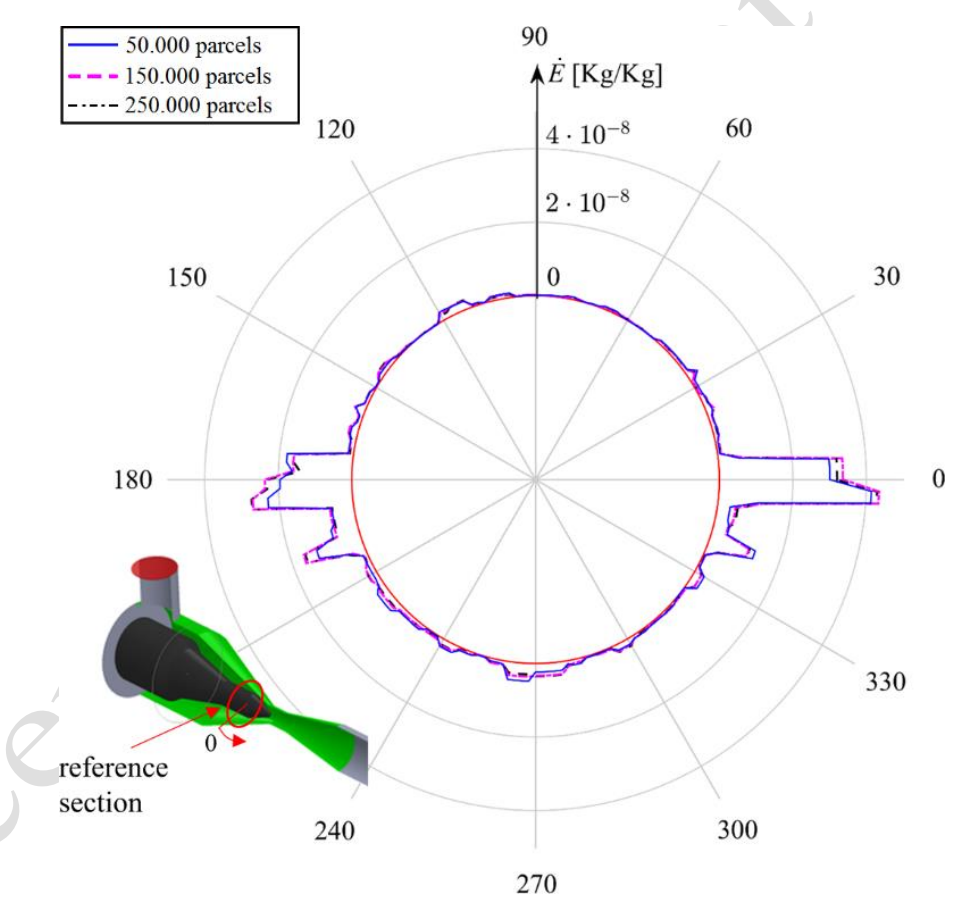

Fig. 6 Effect of the number of tracked parcels on the erosion ratio profile over the circumference depicted at the bottom on the left hand side of the figure.

In order to ensure the consistency of the numerical solution with respect to the number of tracked parcels, the local erosion ratio was determined over the outer wall circumference, depicted in red color at the bottom on the left-hand side in Fig. 6, for three parcel numbers: 50000, 150000, and 250000. In this study, use is made of the 
DNV erosion correlation (Eqs. 18-19) with $n=2.6$ and $K=2.0 \cdot 10^{-9}(\mathrm{~m} / \mathrm{s})^{-n}$. As shown in Fig. 6, the profiles obtained with 150000 and 250000 parcels are very close to each other, and deviate slightly from the 50000 parcels solution. Thus, 150000 parcels were injected in the valve simulations.

\subsection{Validation of multiphase flow modelling}

Validation of the multiphase flow models used in this study was performed by comparison with the experimental data of Okita et al. [5]. The experiments regard the normal impingement of an abrasive jet against a surface and, making reference to the notation introduced in Fig. 2(a), the values of the geometrical quantities are $H=12.7 \mathrm{~mm}, d=8 \mathrm{~mm}$, and $D=76.2 \mathrm{~mm}$. The carrier fluid is water and the jet is submerged, and the average jet velocity at the nozzle exit is $10 \mathrm{~m} / \mathrm{s}$. The solid phase consists of spherical particles with size of $120 \mu \mathrm{m}$ and density of $2700 \mathrm{~kg} / \mathrm{m}^{3}$. The average solid volume fraction at the nozzle exit is $1 \%$.

(a)
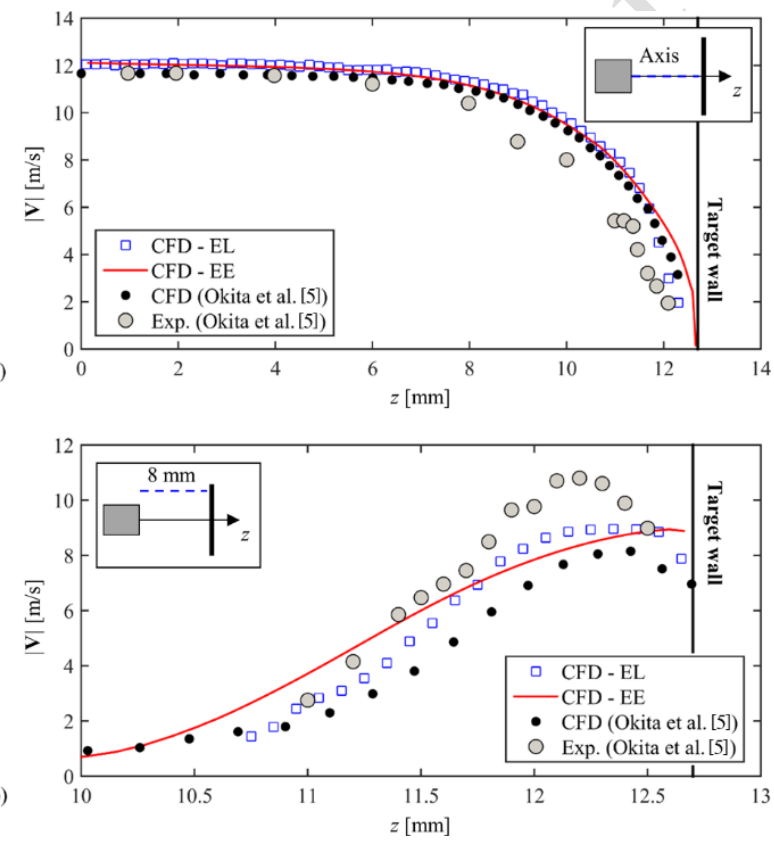

Fig. 7 Validation of two-phase flow modelling

Okita et al. [5] provided PIV measurements of the average particle velocity along the jet axis and $8 \mathrm{~mm}$ far from it. The comparisons are reported in Fig. 7, the horizontal axis of the latter plot being restricted to $z \geq 10$ $\mathrm{mm}$ for the sake of clarity. In the EL computation, the mean particle velocity has been evaluated by dividing the axial size of the domain in subintervals of size $\Delta z=0.1 \mathrm{~mm}$, and then calculating the statistics over the particles transiting through each of them. The overall good agreement between our computations and the 
experiments indicates that the computational models previously described are capable in providing reliable description of the average particle velocity field; particularly, good agreement is observed at the jet axis, whilst for $r=8 \mathrm{~mm}$ CFD agrees with the measurements away from the wall, but it under-predicts the peak in speed close to the wall. A similar behavior has been observed by Okita et al. [5] who used an EL model with a Reynolds Stress Model as turbulence closure. The analysis reported above guarantees the suitability of the used CFD models for the scope of this study, which is focused on an innovative approach which doesn't depend on the specific two-phase models employed.

\section{The mixed Euler-Euler/Euler-Lagrange approach}

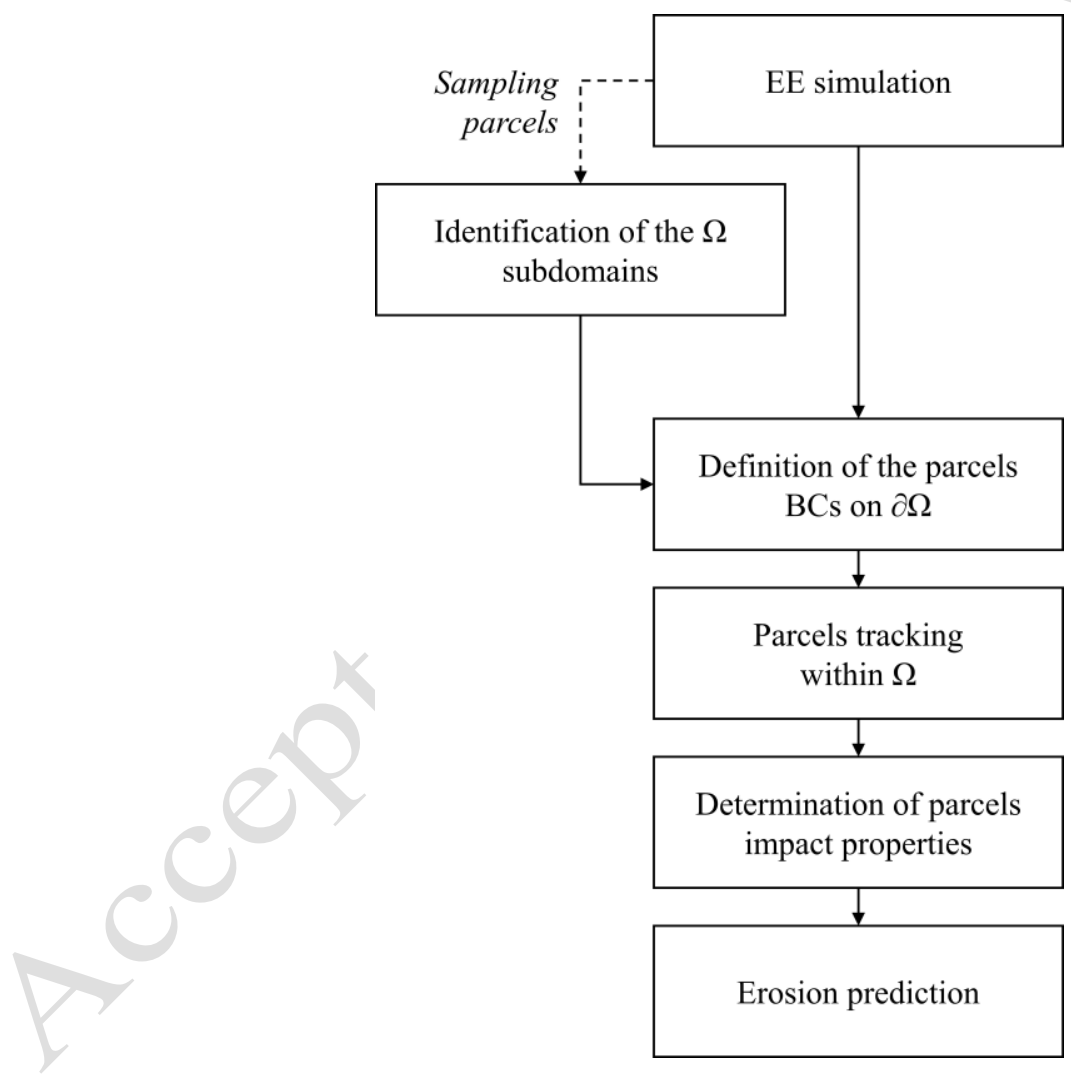

Fig. 8 The mixed EE/EL approach to erosion prediction

The steps to be taken are illustrated in Fig. 8, and they will be now discussed. An EE simulation is performed first, and the average properties of the fluid and the solid phase (i.e. $\mathbf{U}_{E E}, \mathbf{V}_{E E}, P_{E E}, \Phi_{E E}, k_{E E}$, and $\varepsilon_{E E}$ ) are determined in the whole computational domain. Afterwards, the $\Omega$ subdomains are identified as it will be 
discussed later in this section. The parcels trajectories are then determined within $\Omega$ by integrating a system of ordinary differential equations which is analogous to Eq. (1), with the only difference that the fluid properties are obtained from the EE simulation (i.e. $\mathbf{u}_{E L}$ and $P_{E L}$ are replaced by $\mathbf{u}_{E E}$ and $P_{E E}$ respectively; $\mathbf{u}_{E E}$ is again evaluated from the average quantities $\mathbf{U}_{E E}, k_{E E}$, and $\varepsilon_{E E}$ by the stochastic turbulence model of Gosman and Ioannides [36]).

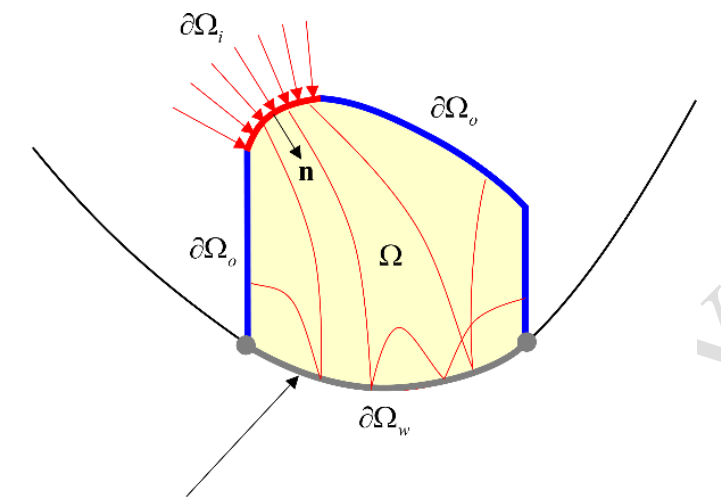

Erosion hotspot location

Fig. 9 A typical $\Omega$ subdomain for a two-dimensional simulation

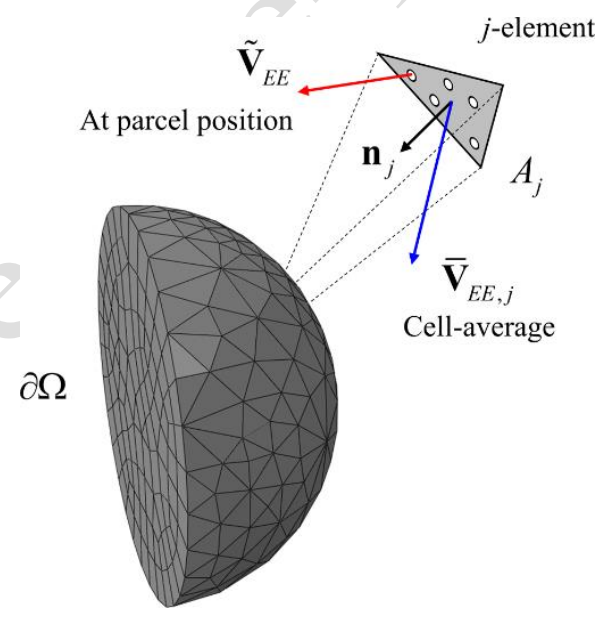

Fig. 10 Triangulation of the boundary of the $\Omega$ subdomain and one surface element.

The border of $\Omega$, referred to as $\partial \Omega$, consists of one or more inlet boundaries $\partial \Omega_{i}$, outlet boundaries $\partial \Omega_{o}$, wall boundaries $\partial \Omega_{w}$ and, possibly, axis of symmetries $\partial \Omega_{a}$ or symmetry planes $\partial \Omega_{s}$ (Fig. 9). After performing a triangulation of the $\partial \Omega$ boundary, each element $j$ is associated a specific boundary condition, i.e. it is established whether $j$ belongs to either $\partial \Omega_{i}, \partial \Omega_{o}, \partial \Omega_{w}, \partial \Omega_{a}$ or $\partial \Omega_{s}$. Particularly, the elements lying on solid walls belong to $\partial \Omega_{w}$ and, there, the normal and tangential components of the reflected parcel velocity are related to 
the corresponding incident values via the normal and tangential restitution coefficients, respectively. In the same way, the elements lying on an axis/plane of symmetry will belong to $\partial \Omega_{a}$ or $\partial \Omega_{s}$, and the parcels bounce with unit restitution coefficients there. The remaining elements will belong to either $\partial \Omega_{i}$ or $\partial \Omega_{o}$, as determined from the outcomes of the EE simulation.

The inlet boundary $\partial \Omega_{i}$ consists of the surface of the $j$ elements in which the EE mass flux enters the domain, that is:

$$
\partial \Omega_{i}=\sum_{j} A_{j} \mid \quad \dot{M}_{E E, j}=A_{j}\left[\rho_{p} \bar{\Phi}_{E E, j}\left(\overline{\mathbf{V}}_{E E, j} \cdot \mathbf{n}_{j}\right)-\frac{\bar{\mu}_{E E, j}^{t}}{\sigma_{\Phi}}\left(\overline{\nabla \Phi_{E E}} \cdot \mathbf{n}_{j}\right)\right]>0
$$

where $A_{j}$ is the area of element $j, \mathbf{n}_{j}$ is the unit normal vector pointing inward, and the over-bar together with the subscript $j$ means that the EE quantities $\Phi_{E E}, \mathbf{V}_{E E}, \mu_{E E}^{t}$, and $\nabla \Phi_{E E}$ have been averaged over $j$. Through each element satisfying the condition (24), a certain number of parcels $N_{j}$ is released as proportional to the EE mass flux entering the domain, that is $N_{j} \sim \dot{M}_{E E, j}$. The positions of the $N_{j}$ parcels are determined from random sampling on a uniform PDE over the element, and their initial velocities are taken from the interpolated values of the mean solid velocity $\mathbf{V}_{E E}$ and the solid turbulent kinetic energy $k_{s, E E}$ at parcels' position, i.e. $\tilde{\mathbf{V}}_{E E}$ and $\tilde{k}_{s, E E}$ respectively (Fig. 10), by means of the following formula:

$$
\mathbf{v}_{P, 0}=\tilde{\mathbf{V}}_{E E}+\xi\left(\frac{2 \tilde{k}_{s, E E}}{3}\right)^{1 / 2}
$$

where, as in Eq. (22), $\xi$ is a three-element vector containing random scalars drawn from the standard PDF. The solid turbulent kinetic energy $k_{s, E E}$ is inferred from the fluid turbulent kinetic energy and the eddy viscosities of the two phases, as follows.

$$
\frac{k_{s, E E}}{k_{E E}}=\frac{\rho_{f}}{\rho_{p}} \frac{\mu_{s, E E}^{t}}{\mu_{E E}^{t}}
$$

The mass flux, which is the same for all parcels, is calculated in respect of the mass conservation principle:

$$
\dot{m}_{P}=\frac{\sum_{j} \dot{M}_{E E, j}}{\sum_{j} N_{j}} \quad j \in \partial \Omega_{i}
$$

The outlet boundary $\partial \Omega_{o}$ consists of the surface of the remaining elements, in which the EE mass flux points outwards, and the parcels simply leaves $\Omega$ on $\partial \Omega_{o}$. 
The definition of the $\Omega$ subdomains is made starting from their wall boundaries $\partial \Omega_{w}$ which are the surfaces whose erosion behavior is to be investigated. These surfaces are likely to contain the erosion hotspots locations, i.e. the parts of the pipeline component which are most vulnerable to wear. In many applications, their identification may not be an issue since they could be already known on the basis of experience. However, if no information is available at all, gross localization of the surfaces most exposed to wear can be identified by tracking a small number of sampling parcels within the entire flow domain after performing the EE simulation. It is worth notice that this calculation will have little effect on the computational gain of using the proposed method; in fact, the high computational burden of the EL computations mainly arise from the considerable number of trajectories necessary to achieve reliable statistics, but only a limited number of trajectories (around 1000 in our simulations, but this value is expected to be problem-dependent) are needed to understand with sufficient accuracy the erosion hotspots locations. Once they are identified, and so the $\partial \Omega_{w}$ boundaries are, the $\Omega$ subdomains can be defined, bearing in mind that the smaller their size is, the greater the reduction of the computational burden is. However, the definition of $\partial \Omega_{i}$ and $\partial \Omega_{o}$ as previously reported is consistent only if all "real" particles cross each element in the same direction, i.e. the particles crossing the elements belonging to $\partial \Omega_{i}$ must all enter the domain and those crossing the elements belonging to $\partial \Omega_{o}$ must all leave the domain. The release of sampling parcels helps in understanding whether this condition is satisfied, as it will be shown in the application tests.

\section{Application}

The mixed EE/EL approach has been applied to the two test cases sketched in Fig. 2, namely the normal impingement of an abrasive jet against a surface, and a needle and seat choke valve. The outcomes of these analyses are reported in subsections 4.1 and 4.2, respectively. As already noticed, we validated the new approach against the standard EL-based predictive practice, which, particularly when applied to complex geometries, is actually dependent on the following restrictive hypothesis: (1) low particle loading, so that the assumption of "one way coupling" regime holds; (2) short exposure to wear, so that geometry changes due to erosion are negligible. The results obtained at this stage will open the way for removing these hypotheses in practical applications. 


\subsection{Abrasive jet impingement}

Table 1 Constants in the E/CRC erosion correlation (Eqs. 15-17) employed in the abrasive jet test, as determined by Okita et al. [5] for the same flow condition (sand particles with $d_{p}=300 \mu \mathrm{m}$; target material: Aluminium 6061).

\begin{tabular}{cc}
\hline Parameter & Value \\
\hline$H_{v}(\mathrm{GPa})$ & 1.12 \\
$F_{s}$ & 1.00 \\
$C$ & $3.28 \mathrm{E}-07$ \\
$n$ & 2.41 \\
$n 1$ & 0.50 \\
$n 2$ & 2.50 \\
$n 3$ & 0.50 \\
$f$ & 2.19 \\
\hline
\end{tabular}

In this first validation study we reproduced some lab experiments performed by Okita et al. [5] concerning the normal impingement of an abrasive sand-water submerged jet against a specimen of Aluminium 6061. The values of the geometrical quantities are the same of the earlier flow validation study, namely $H=12.7 \mathrm{~mm}, d=8$ $\mathrm{mm}$, and $D=76.2 \mathrm{~mm}$ (Fig. 2a). In the same way, also the average jet velocity and the average solid volume fraction are kept the same, i.e. $10 \mathrm{~m} / \mathrm{s}$ and $1 \%$ respectively. However, in this test the particle size is increased to $300 \mu \mathrm{m}$ leaving the density unchanged $\left(2700 \mathrm{~kg} / \mathrm{m}^{3}\right)$. In fact, Okita et al. [5] provided erosion ratio measurements for this kind of particles, and determined the values of the constants of the E/CRC erosion correlation (Eqs. 15-17) for this flow conditions. This correlation has been used together with the constants reported by the experimenters, listed in Table 1 ; the density of the target material was taken as $2700 \mathrm{~kg} / \mathrm{m}^{3}$. The ratio between the nozzle-to-specimen distance $H$ and the jet velocity at the nozzle axis was taken as the characteristic fluid time scale $\tau_{f}$, yielding a Stokes number of 8.2.

Details of the numerical setup, including flow domain, boundary conditions, and computational methodology, have already been given in Section 2. After simulating the two-phase flow by means of the EE model, we determined the $\Omega$ subdomain. This was done by releasing 1000 sampling parcels as in a full EL simulation, the only difference being that, when integrating Eq. (1), the fluid properties where determined from the EE model instead of the single-phase RANS. Application of the methodology for erosion prediction described in Subsection 2.5 to the sampling parcels allowed achieving gross estimation of the portion of the specimen most vulnerable to wear, thereby determining the wall boundary of the $\Omega$ subdomain and, in cascade, the subdomain itself. Figure 11(a) shows the local erosion ratio $\dot{E}[\mathrm{Kg} / \mathrm{Kg}]$ distribution as obtained from the 
injection of 1000 sampling parcels, whilst Figure 11(b) reports 100 representative trajectories and the $\Omega$ subdomain as defined based on these results, together with its boundaries $\partial \Omega_{i}$ (inlet), $\partial \Omega_{o}$ (outlet), $\partial \Omega_{w}$ (wall), and $\partial \Omega_{a}$ (axis of symmetry). From Fig. 11(b) it is evident that $\partial \Omega_{i}$ and $\partial \Omega_{o}$ are crossed only by parcels entering and leaving the domain, respectively, thereby confirming the consistent definition of the boundary conditions on these borders.
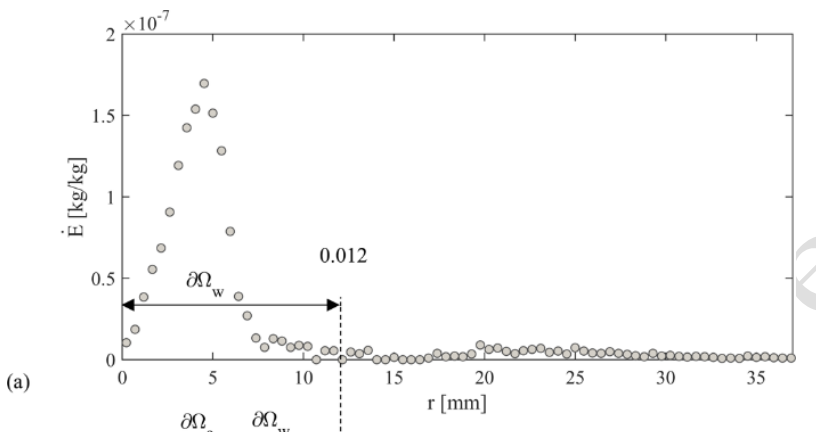

(b)

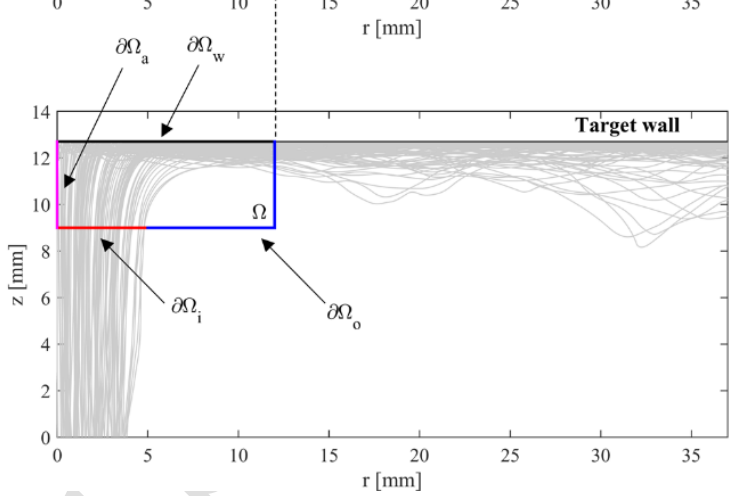

Fig. 11 The erosion ratio profile as obtained from 1000 sampling parcels (a) and the trajectories of 100 out of them (b) for the identification of the $\Omega$ subdomain

In order to guarantee the consistency of the proposed approach, we compared the average properties of the solid phase on the inlet boundary $\partial \Omega_{i}$ as obtained from the EL and EE simulations. Particularly, we focused on the modulus of the mean velocity of the solid phase $|\mathbf{V}|$ and the local solid mass flux $\dot{m}_{s}$, since the compatibility of the estimates of these parameters is fundamental to assure that the parcels boundary conditions on $\partial \Omega_{i}$ have been correctly imposed. This is proved from the results shown in Fig. 12, where the similarity between the EL and EE solutions on $\partial \Omega_{i}$ is evident. The statistics have been evaluated after dividing the boundary $\partial \Omega_{i}$ in subintervals of size $\Delta r=0.25 \mathrm{~mm}$. 
(a)

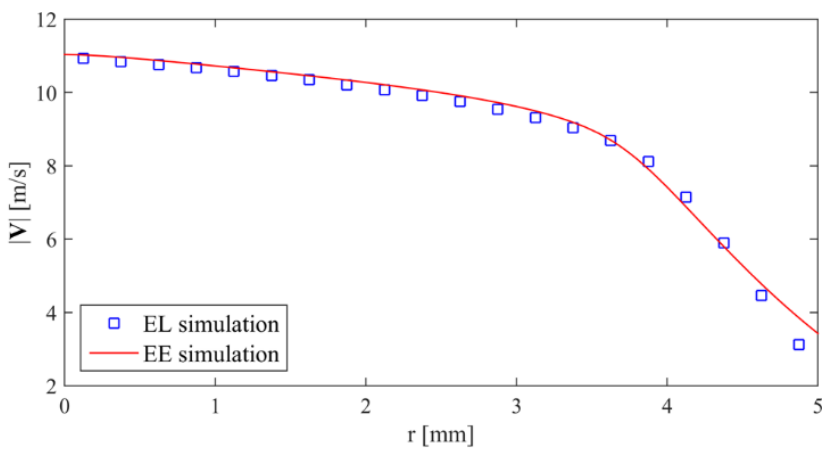

(b)

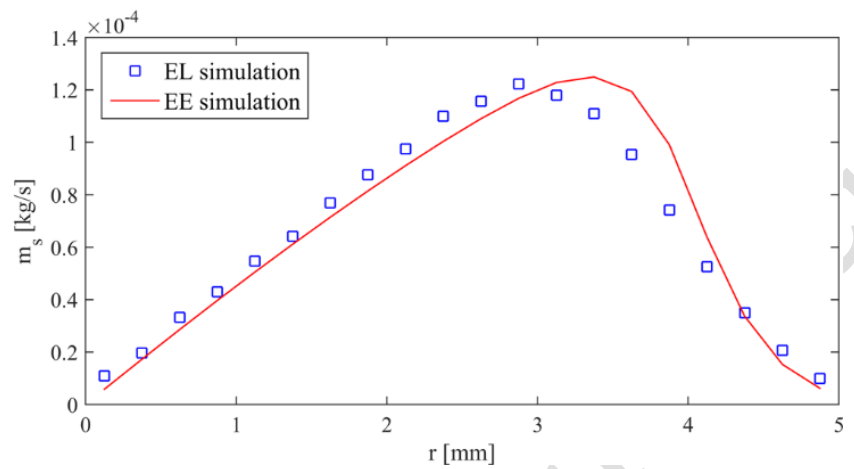

Fig. 12 Comparison between EL and EE solutions at the inlet boundary of the $\Omega$ subdomain: (a) modulus of the mean velocity of the solid phase; (b) local solid mass flux.

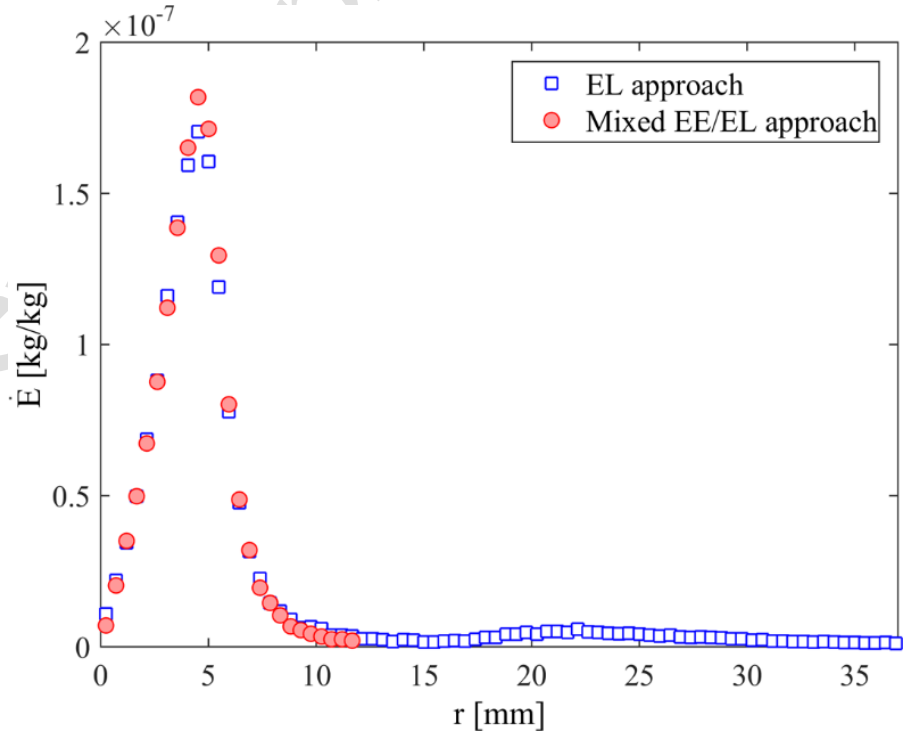

Fig. 13 Radial distribution of the erosion ratio as obtained from the standard EL-based approach and the mixed EE/EL approach. 
After performing the tracking of the parcels within $\Omega$, we determined the local erosion ratio profile along the radial coordinate, and compare it against the EL solution. The results, shown in Fig. 13, evidence the substantial coincidence of the two distributions, thereby confirming the reliability of the mixed EE/EL approach. The integral erosion ratios are $1.54 \cdot 10^{-6} \mathrm{~kg} / \mathrm{kg}$ and $1.40 \cdot 10^{-6} \mathrm{~kg} / \mathrm{kg}$ with the EL approach and the $\mathrm{EE} / \mathrm{EL}$ approach, respectively; this indicates that, if $\Omega$ is properly defined in relation to the location of the erosion hotspots, tracking the parcels' trajectories within $\Omega$ only does not alter significantly the integral erosion ratio estimates. Moreover, the two obtained values show fairly good agreement with the experimental result, which is equal to $(3.21 \pm 0.44) \cdot 10^{-6} \mathrm{~kg} / \mathrm{kg}$.

Compared to the EL approach, the proposed one allows considerable reduction in the number of solved flow variables, which is calculated as follows: EL approach $=P_{E L}, \mathbf{U}_{E L}, k_{E L}$, and $\varepsilon_{E L}$ in each computational cell $+\mathbf{x}_{E L}$ and $\mathbf{v}_{E L}$ for each parcel at each Lagrangian time instant; mixed EE/EL approach $=P_{E E}, \mathbf{U}_{E E}, \mathbf{V}_{E E}, \Phi_{E E}$ $k_{E E}$, and $\varepsilon_{E E}+\mathbf{x}_{E L}$ and $\mathbf{v}_{E L}$ for each parcel at each Lagrangian time instant within the $\Omega$ subdomain. For this simple $2 \mathrm{D}$ axisymmetric case, the reduction is from about $9.70 \cdot 10^{7}$ to about $2.50 \cdot 10^{7}$, corresponding to about $75 \%$ less (actually, in both cases almost all of the calculated data are parcels' characteristics in the Lagrangian framework).

Estimating the gain in CPU time is more difficult, since the calculation time is strongly dependent upon the used machine as well as the employed solution algorithm and the level of parallelization. Just to give an idea, we report the data that we obtained on a machine with Intel Core i7 CPU at $2.80 \mathrm{GHz}$ and $8 \mathrm{~GB}$ RAM. Switching from a single-phase to a EE simulation increases the simulation time from about 20 to about 26 minutes (30\%) but, at the same time, following the parcels' trajectories within $\Omega$ instead of the whole domain reduces the time for particle tracking and erosion ratio determination from about 36 to about 16 minutes (-55\%). The total simulation time is thus reduced from about $56^{\prime}$ to about $40^{\prime}$, the gain being around $30 \%$.

\subsection{Needle and seat valve}

The second validation test case concerns the needle and seat choke valve sketched in Fig. $2 \mathrm{~b}$. The flow conditions, in terms of flow velocity and composition of the mixture, have already been reported in Subsection 2.5 , namely: mean flow velocity $=7 \mathrm{~m} / \mathrm{s}$, particle density $2700 \mathrm{~kg} / \mathrm{m}^{3}$, particle diameter $=400 \mu \mathrm{m}$; and solid volume fraction $=1 \%$. The device is assumed made of stainless steel SUS304 $\left(\rho_{w}=7929 \mathrm{~kg} / \mathrm{m}^{3}\right)$; in the first part of the analysis, the used erosion correlation is the DNV one (Eq. 18-19), and the values of the numerical 
constants $n$ and $K$ are those recommended for this material, namely 2.6 and $2.0 \cdot 10^{-9}(\mathrm{~m} / \mathrm{s})^{-2.6}$ respectively [19]. The characteristic fluid time scale $\tau_{f}$ was taken as the ratio between the diameter of the inlet pipe and the mean flow velocity at the inlet section, resulting in a Stokes number of about 1.7.

(a)
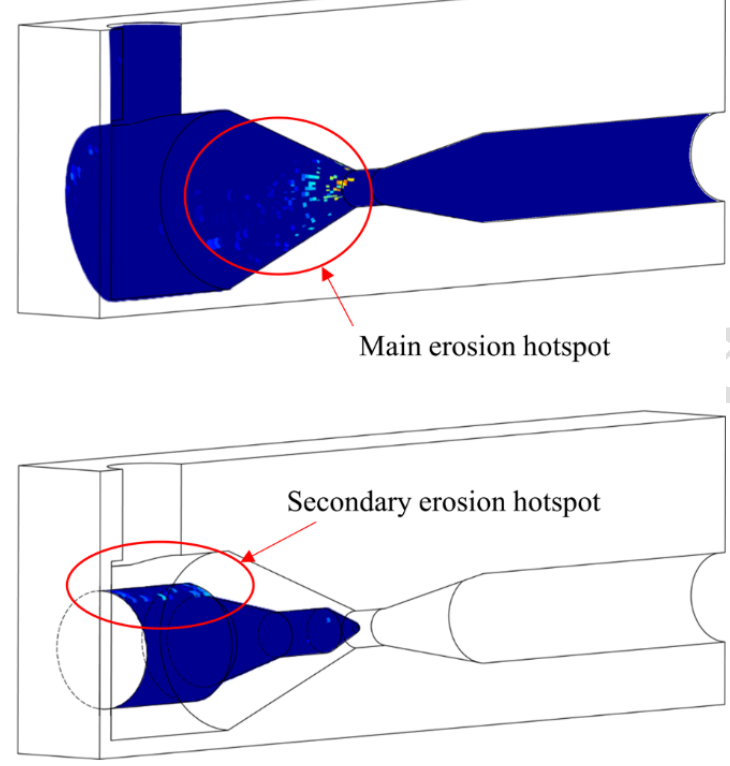

(b)

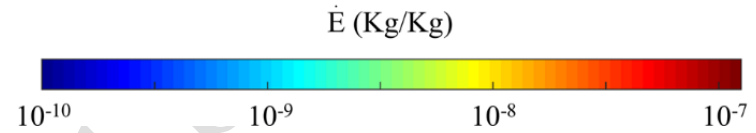

Fig. 14 Local erosion ratio distribution on the valve walls as obtained from 1000 sampling parcels. The DNV erosion correlation (Eqs. 18-19) with the constants recommended for steel grades is used.

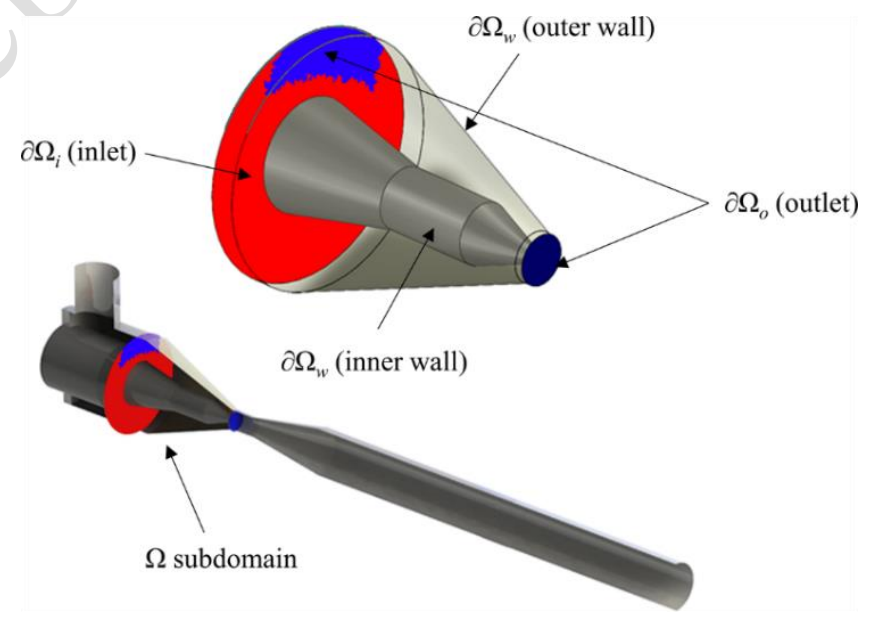

Fig. 15 The $\Omega$ subdomain and its boundaries. 

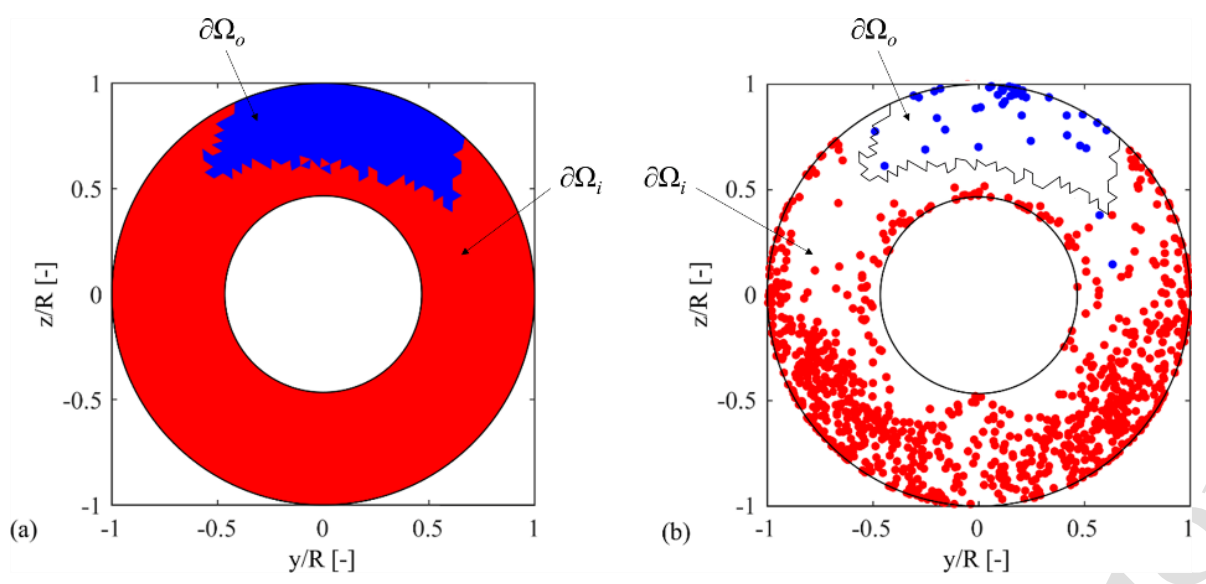

Fig. 16 Upstream face of the $\Omega$ subdomain: (a) identification of the elements belonging to the inlet (red) and the outlet (blue) boundaries based on the EE solid mass flux; (b) positions in which the trajectories of 1000 sampling parcels cross the face. Red and blue points indicate that the parcels are entering the leaving the domain, respectively.

An EE simulation of the liquid-solid flow within the valve was performed first, and 1000 sampling parcels were released at the inlet section, producing the local erosion ratio distribution shown in Fig. 14. The results clearly indicates the existence of two erosion hotspots, the most severe being in correspondence to the reducer upstream the seat and the other one caused by the direct impingements of the incoming particles. The $\Omega$ subdomain has been defined in order to finalize the mixed EE/EL approach to the prediction of the erosion of the reducer of the valve, and it is shown in Fig. 15. The surface of the $\Omega$ subdomain, $\partial \Omega$, has been triangulated and its boundaries $\partial \Omega_{w}, \partial \Omega_{o}$, and $\partial \Omega_{i}$ determined following the procedure reported in Section 3. Particularly, the downstream face of the subdomain is an outlet, the side faces are wall boundaries, whilst the upstream face consists of both inlet and outlet, as shown in Figs. 15 and 16(a). The analysis of the trajectories of the 1000 sampling parcels confirms that $\partial \Omega_{i}$ and $\partial \Omega_{o}$ have been consistently defined. In fact, Fig. 16(b) shows the locations in which each sampling parcel intersect the upstream face of the subdomain; red and blue points indicate that the parcels are entering and leaving the domain, respectively. Except for a couple of parcels out over 1000, all the parcels passing through $\partial \Omega_{i}$ actually enter the domain and, similarly, all those passing through $\partial \Omega_{o}$ actually leave it.

As done in the abrasive jet case, we compared the EL and EE solutions on the inlet boundary $\partial \Omega_{i}$ in order to guarantee the reliability of the parcels boundary conditions as obtained from the output of an EE simulation. Particularly, we focused on the mean velocity of the solid phase and the local solid mass flux. The comparison 

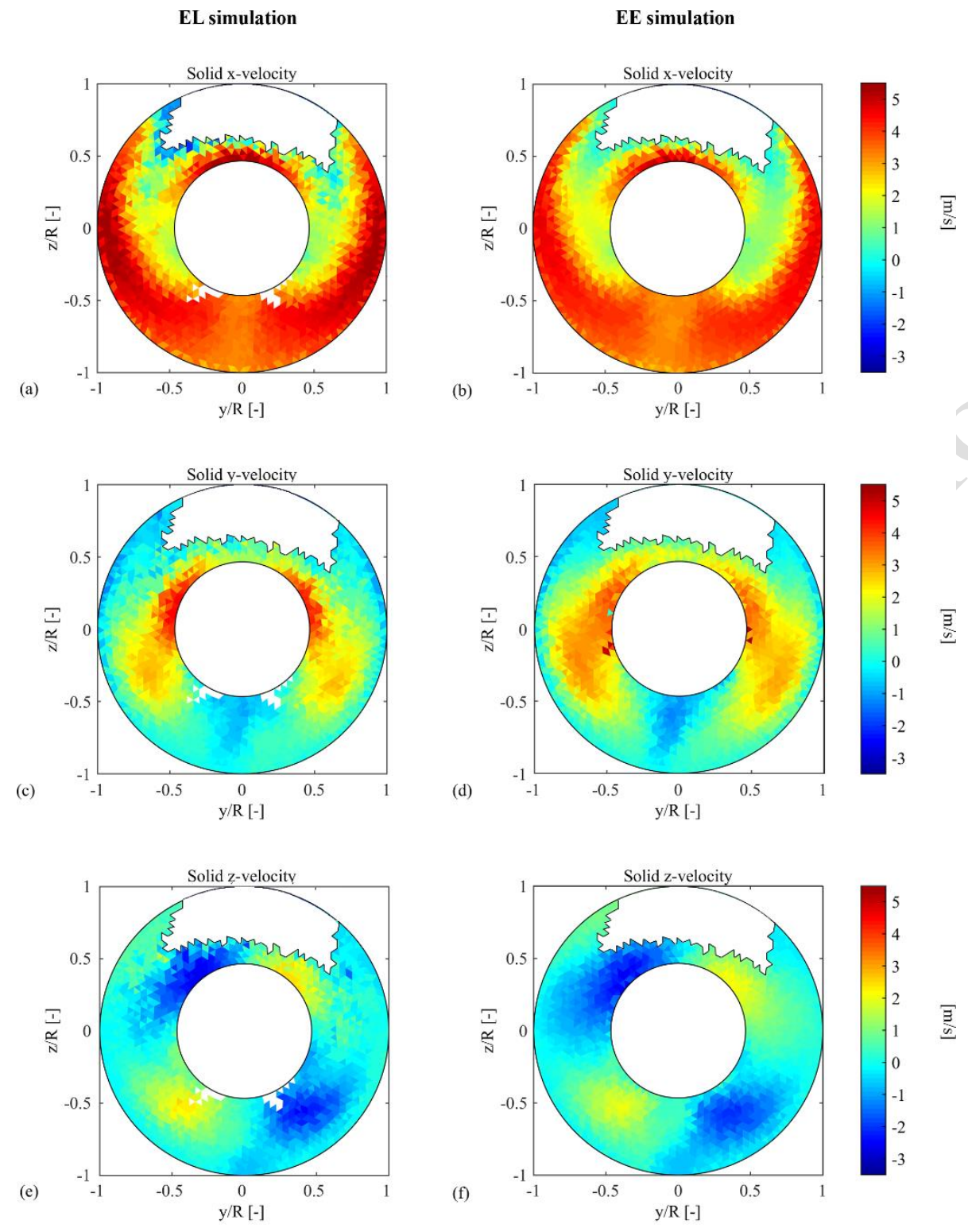

(f)

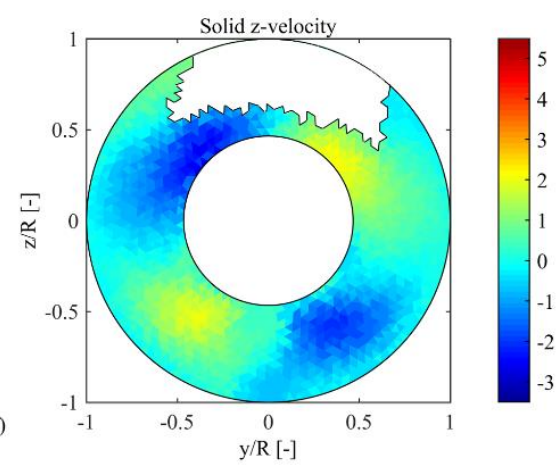

曐

疍

$\underset{\Xi}{\Xi}$
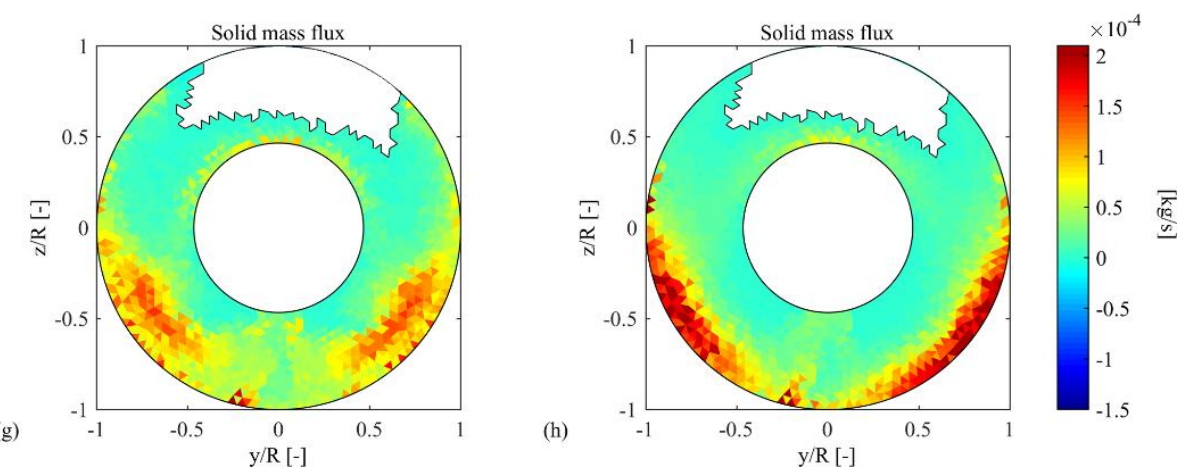

Fig. 17 Comparison between the EL solution (on a sample of 70000 parcels) and EE one on the inlet boundary of the $\Omega$ subdomain, in terms of the three components of the mean velocity of the solid phase, and the local solid mass flux. 
is shown in Fig. 17, and it evidences the compatibility between the two CFD solutions also in the valve case. It is worth noticing that the statistics of the EL solution have been evaluated on a sample of 70.000 parcels only for reasons of computational burden.

Mixed EE/EL approach

(a)

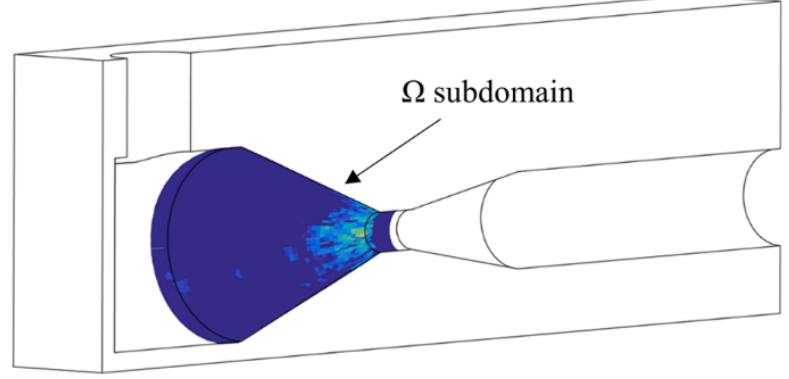

EL simulation

(b)
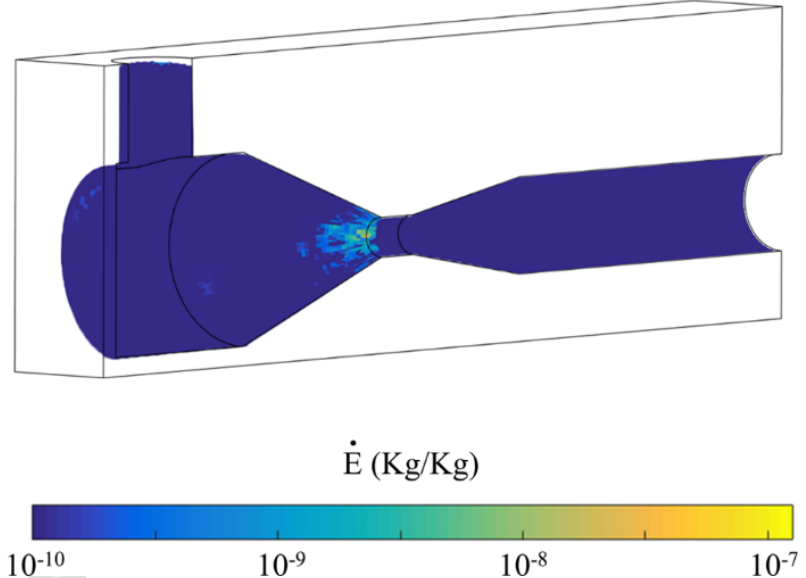

Fig. 18 (a) Local erosion ratio distribution on the wall boundary of the $\Omega$ subdomain; (b) Local erosion ratio distribution on the outer wall of the valve chamber as obtained by a full EL simulation.

The local erosion ratio distribution on the wall boundary of the $\Omega$ subdomain is calculated and depicted in Fig. 18(a), revealing analogy with the EL solution, shown in Fig. 18(b). In order to provide a more quantitative comparison between the two solutions, Fig. 19 shows the local erosion ratio profiles on a circumference located nearly at the end of the reducer, where the maximum wear occurs. The reference section is shown at the bottom on the left hand side of the figure. Moreover, Table 2 compares the integral erosion ratios as obtained by integration of the local quantities over the reducer. The post-processing analysis has been repeated by employing the Oka erosion correlation (Eqs. 20-21) with the numerical constants used by Nguyen et al. [6] for 
stainless steel SUS304, reported in Table 3. The deviation of a factor 3 between the integral wear ratios confirms the significant influence of the used erosion correlation in erosion prediction analyses.

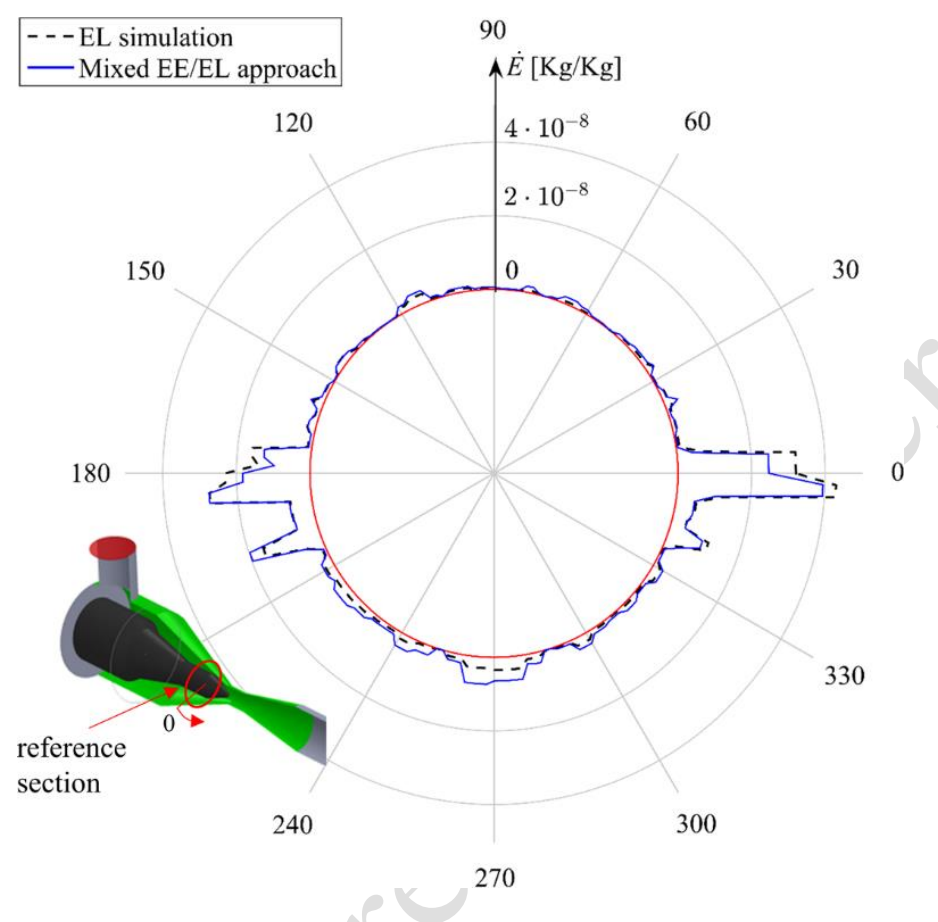

Fig. 19 Polar plot representing the local erosion ratio distribution over the circumference depicted at the bottom on the left hand side of the figure, when the predicted maximum wear occurs. Comparison between the mixed EE/EL approach and a full EL simulation.

Table 2 Integral erosion ratios of the reducer as obtained from the EL approach and the mixed EE/EL approach with the $\Omega$ subdomain depicted in Fig. 15. The estimates are obtained by applying two erosion correlations, namely the DNV one (Eqs. 18-19) and the Oka one (Eqs. 20-21) with the numerical constants reported in Table 3.

\begin{tabular}{ccc}
\hline & \multicolumn{2}{c}{ Integral erosion ratio on reducer $(\mathrm{Kg} / \mathrm{Kg})$} \\
\hline Approach & DNV correlation & Oka correlation \\
\hline EL & $6.41 \cdot 10^{-7}$ & $1.74 \cdot 10^{-6}$ \\
Mixed EE/EL & $7.46 \cdot 10^{-7}$ & $2.04 \cdot 10^{-6}$ \\
\hline
\end{tabular}


Table 3 Constants in the Oka erosion correlation (Eqs. 20-21) employed in the valve case, as reported by Nguyen et al. [6] for sand particles and stainless steel SUS304 as target material.

\begin{tabular}{cc}
\hline Parameter & Value \\
\hline$H_{v}(\mathrm{GPa})$ & 1.96 \\
$\rho_{w}$ & $7929 \mathrm{~kg} / \mathrm{m}^{3}$ \\
$V^{\prime}$ & $104 \mathrm{~m} / \mathrm{s}$ \\
$D^{\prime}$ & $326 \mu \mathrm{m}$ \\
$k 1$ & -0.112 \\
$k 3$ & 0.19 \\
$K$ & 65 \\
$s 1$ & 0.71 \\
$s 2$ & 2.4 \\
$q 1$ & 0.14 \\
$q 2$ & -0.94 \\
\hline
\end{tabular}

For the reasons already stated, there are no erosion ratio measurements which can be used for the experimental validation of our numerical prediction. However, comparison was made between the computed integral erosion ratios reported in Table 2 and the predicted value obtained by application of the empirical formula reported in the DNV Recommended Practice [20] for erosion ratio in a flow reducer. This comparison should be done with caution because of the greater complexity of the flow in presence of the needle compared to that in a reducer connecting two straight pipes. However, in the absence of more appropriate formulas, the DNV model can provide at least a very rough estimate of the erosion ratio in the valve reducer. The DNV model is as follows:

$$
\dot{E}_{\text {red }}=K \cdot F(\alpha) \cdot U^{n} \cdot\left(1-\frac{d_{2}^{2}}{d_{1}^{2}}\right) \cdot \min \left(\frac{10^{6} \cdot d_{p}}{30 \sqrt{\rho_{f}}}, 1.0\right)
$$

where: $K$ and $n$ are the numerical constants of the DNV erosion correlation (Eq. 18) and, similarly, the angle function $F$ is given by Eq. (19); $\alpha$ is a characteristic particle impact angle which, if unknown, is suggested to be set equal to $60^{\circ}$; $U$ is the average fluid velocity after the contraction; and $d_{1}$ and $d_{2}$ are the diameter after and before the reducer, respectively. Application of Eq. (24) to the valve case yields $\dot{E}_{\text {red }}=1.24 \cdot 10^{-6} \mathrm{Kg} / \mathrm{Kg}$, which is compatible with the computed values reported in Table. 2.

Some considerations about the gain of using the mixed EE/EL approach instead of the EL are provided, making reference to the reduction in both the number of solved quantities as defined at the end of Subsection 4.1 and the simulation time. The number of solved quantities drops from $8.2 \cdot 10^{8}$ to $5.1 \cdot 10^{8}$, about $37 \%$ lower (again, almost all of these quantities are parcels' positions and velocities in the Lagrangian framework). On a 
machine with Intel Core i7 CPU at $2.80 \mathrm{GHz}$ and $8 \mathrm{~GB}$ RAM, running an EE simulation of the valve instead of a single-phase one increases the computer time from about $18 \mathrm{~h}$ to about $21 \mathrm{~h}(+17 \%)$. However, limiting the particle tracking to the $\Omega$ subdomain results in a reduction of the computer time for particle tracking and erosion ratio calculation from about 14.8 to $5.6 \mathrm{~h}(-63 \%)$. Summing up the two contributions, the total simulation time is reduced of about $13 \%$.

The data reported above, as well as those at the end of the previous subsection, indicate that, at the expense of a limited increase in the computational burden for flow computation, the mixed EE/EL approach allows a very significant reduction of the computational burden for particle tracking and erosion ratio determination (for the abrasive jet and the needle and seat valve, respectively: $-75 \%$ and $-37 \%$ in terms of calculated parcels' positions and velocities, $-55 \%$ and $-63 \%$ in terms of CPU time). The overall gain is expected to become even more significant outside the assumption of "one-way coupling" regime and when geometry changes due to erosion are accounted for, because in these cases the parcels' trajectories have to be iteratively recalculated during the simulation. This will open the way for addressing practical application cases involving dense flows or long exposures to erosion.

\section{Conclusion}

In the oil\&gas industry, the prediction of erosion of pipeline components subjected to dense flows or long exposures to aggressive flow conditions which cause modifications to their geometry is actually prevented due to the excessive computational burden of the available approach to erosion prediction, which relies on EulerLagrange (EL) simulations. In this paper, we propose an alternative approach, referred to as mixed EulerEuler/Euler-Lagrange (EE/EL), in which the Lagrangian tracking of the parcels' trajectories is performed only within certain subdomains adjacent to the surfaces which have been selected as the most vulnerable to erosion, and the fluid flow field within the subdomains and the conditions to be applied to the parcels on the boundaries of the subdomains are obtained from an Euler-Euler (EE) simulation (Section 3).

The mixed EE/EL approach has been applied to two test cases with increasing complexity, namely an abrasive jet impingement test and a needle and seat choke valve (Fig. 2). The new approach has been compared against the standard EL-based approach, thereby under the hypotheses which make the latter practically applicable, namely: (1) low solid loading so that the assumption of "one-way coupling" regime holds; (2) short erosion time, so that geometry changes due to erosion are negligible. 
After verifying the consistency of the fluid dynamic conditions imposed at the boundaries of the subdomains (Figs. 11, 12, 16, and 17), we proved that the mixed EE/EL approach produces similar erosion ratio estimates as the EL-based one (Figs. 13, 18, 19, and Table 2), and compatible with the available experimental measurements as well as simplified models of practical utility. Moreover, it allows very significant reduction of the computational burden for particle tracking and wear estimation, thereby increasing the possibility of gathering reliable wear predictions in practical applications.

\section{References}

[1]. R.J.K. Wood, T.F. Jones, N.J. Miles, J. Ganeshalingam, Upstream swirl-induction for reduction of erosion damage from slurries in pipeline bends, Wear 250 (2001), 770-778.

[2]. E.J. Wentzel, C. Allen, Erosion-corrosion resistance of tungsten carbide hard metals with different binder compositions, Wear 181-183 (1995), 63-69.

[3]. V.A. Pugsley, C. Allen, Microstructure/property relationships in the slurry erosion of tungsten carbidecobalt, Wear 225-229 (1999), 1017-1024.

[4]. Y. Zhang, E.P. Reuterfors, B.S. McLaury, S.A. Shirazi, E.F. Rybicki, Comparison of computed and measured particle velocities and erosion in water and air flows, Wear 263 (2007), 330-338.

[5]. R. Okita, Y. Zhang, B.S. McLaury, S.A. Shirazi, Experimental and Computational Investigations to Evaluate the Effects of Fluid Viscosity and Particle Size on Erosion Damage, ASME J. Fluids Eng. 134 (2012), 061301.

[6]. V.B. Nguyen, Q.B. Nguyen, Z.G. Liu, S. Wan, C.Y.H. Lim, Y.W. Zhang, A combined numerical experimental study on the effect of surface evolution on the water sand multiphase flow characteristics and the material erosion behavior, Wear 319 (2014), 96-109.

[7]. Q.B. Nguyen, V.B. Nguyen, C.Y.H. Lim, Q.T. Trinhm S. Sankaranarayanan, Y.W. Zhang, M. Gupta, Effect of impact angle and testing time on erosion of stainless steel at higher velocities, Wear 321 (2014), 87-93.

[8]. B. Sun, J.C. Fan, D. Wen, Y.Y. Chen, An experimental study of slurry erosion involving tensile stress for pressure pipe manifold, Tribol. Int. 82 (2014), 280-286. 
[9]. A. Mansouri, H. Arabnejad, S.A. Shirazi, B.S. McLaury, A combined CFD/experimental methodology for erosion prediction, Wear (2014), http://dx.doi.org/10.1016/j.wear.2014.11.025i

[10]. J.S. Mason, B.V. Smith, The Erosion of Bends by Pneumatically Conveyed Suspensions of Abrasive Particles, Powder Technol. 6 (1972), 323-335.

[11]. J. Postlethwaite, S. Nesic, Erosion in Disturbed Liquid/Particle Pipe Flow: Effects of Flow Geometry and Particle Surface Roughness, Corrosion 49 (1993), 850-857.

[12]. X. Chen, B.S. McLaury, S.A. Shirazi, Application and experimental validation of a computational fluid dynamics (CFD)-based erosion prediction model in elbows and plugged tees, Comput. Fluids 33 (2004), 1251-1272.

[13]. R. Haugen, O. Kvernvold, A. Ronold, R. Sandberg, Sand erosion of wear-resistant materials: Erosion in choke valves, Wear 186-187 (1995): 179-188.

[14]. L. Nokleberg, T. Søntvedt, Erosion of oil\&gas industry choke valves using computational fluid dynamics and experiment, Int. J. Heat Fluid Flow 19 (1998), 636-643.

[15]. M.S. Wallace, W.M. Dempster, T. Scanlon, J. Peters, S. McCulloch, Prediction of impact erosion in valve geometries, Wear 256 (2004), 927-936.

[16]. D.W. Wheeler, R.J.K. Wood, D. Harrison, E. Smith, Application of diamond to enhance choke valve life in erosive duties, Wear 261 (2006), 1087-1094.

[17]. E. Gharaibah, Y. Zhang, R. Paggiaro, J. Friedemann, Prediction of Sand Erosion in Choke Valves - CFD Model Development and Validation against Experiments, Proc. OTC Offshore Technology Conference (2013), Paper No. OTC 24271.

[18]. R.W. Lyczkowski, J.X. Bouillard, State-of-the-art review of erosion modelling in fluid/solid systems, Prog. Energy Comb. Sci. 28 (2002), 543-602.

[19]. M. Parsi, K. Najmi, F. Najafifard, S. Hassani, B.S. McLaury, S.A. Shirazi, A comprehensive review of solid particle erosion modeling for oil and gas wells and pipelines applications, J. Nat. Gas Sci. Eng. 21 (2014), 850-873.

[20]. Det Norske Veritas. Recommended Practice RP O501. Erosive Wear in Piping Systems. Revision 4.2 (2007).

[21]. Y.I. Oka, H. Ohnogi, T. Hosokawa, M. Matsumura, The impact angle dependence of erosion damage caused by solid particle impact, Wear 203-204 (1997), 573-579. 
[22]. Y.I. Oka, K. Okamura, T. Yoshida, Practical Estimation of Erosion Damage caused by Solid Particle Impact, Part 1: Effects of Impact Parameters on a Predictive Equation, Wear 259 (2005), 95-101.

[23]. Y. Zhang, B.S. McLaury, S.A. Shirazi, Improvements of Particle Near-Wall Velocity and Erosion Prediction Using a Commercial CFD Code, ASME J. Fluids Eng. 131 (2009), 031303.

[24]. D.O. Njobuenwu, M. Fairweather, Modelling of pipe bend erosion by dilute particle suspensions, Comput. Chem. Eng. 42 (2012), 235-247.

[25]. G.C. Pereira, F.J. de Souza, D.A. de Moro Martins, Numerical prediction of the erosion due to particles in elbows, Powder Technol. 261 (2014), 105-117.

[26]. E. Loth, Numerical approaches for motion of dispersed particles, droplets and bubbles, Prog. Energy Comb. Sci. 26 (2000), 161-223.

[27]. E. Loth, Particles, drops and bubbles: fluid dynamics and numerical methods. Draft for Cambridge University Press, 2011. To be published.

[28]. L. Guomei, W. Yueshe, H. Renyang, C. Xuewen, L. Changzhi, M. Tao, Numerical simulation of predicting and reducing solid particle erosion of solid-liquid two-phase flow in a choke, Pet. Sci. 6 (2009), 91-97.

[29]. G.V. Messa, M. Malin, S. Malavasi, Numerical prediction of fully-suspended slurry flow in horizontal pipes, Powder Technol. 256 (2014), 61-70.

[30]. G.V. Messa, S. Malavasi, Improvements in the numerical prediction of fully-suspended slurry flow in horizontal pipes, Powder Technol. 270 (2015), 358-367.

[31]. G.V. Messa, S. Malavasi, Numerical prediction of dispersed turbulent liquid-solid flows in vertical pipes, J. Hydraul. Res. 52(5) (2014), 684-692.

[32]. A. Forder, M. Thew, D. Harrison, A numerical investigation of solid particle erosion experienced by oilfield control valves, Wear 216 (1998), 184-193.

[33]. H.K. Versteeg, W. Malalasekera, An Introduction to Computational Fluid Dynamics - The Finite Volume Method, Pearson Prentice Hall, Harlow UK, 2007.

[34]. B.E. Launder, D.B. Spalding, The numerical computation of turbulent flows, Comput. Meth. Appl. Mech. Eng. 3 (1974), 269-289.

[35]. L. Shiller, A. Naumann, A drag coefficient correlation, Z. Ver. Deutsch. Ing. 77 (1935), 318-320.

[36]. A.D. Gosman, E. Ioannides, Aspects of Computer Simulation of Liquid-Fueled Combustors, J. Energy 7 (1981), 482-490. 
[37]. D.B. Spalding, Numerical computation of multi-phase fluid flow and heat transfer, in: C. Taylor, K. Morgan (Eds.), Recent Advances in Numerical Methods in Fluids, Pineridge Press Limited, Swansea, UK, 1980, pp. 139-168.

[38]. D.B. Spalding, Mathematical Modelling of Fluid-Mechanics, Heat-Transfer and Chemical-Reaction Processes: A Lecture Course, CFDU Report HTS/80/1, Imperial College, London, 1980.

[39]. D.B. Spalding, PHOENICS: a General-Purpose Computer Program for Multi-Dimensional One- and TwoPhase Flow, CFDU Report HTS/81/11, Imperial College, London, 1981.

[40]. J.O. Hinze, Turbulence, McGraw-Hill, New York, 1975.

[41]. S. Malavasi, G.V. Messa, CFD Modelling of a choke valve under critical working conditions, Proc. ASME 2014 Pressure Vessel and Piping Conference ASME PVP2014 (2014), Paper No. 28629.

[42]. H. Schlichting, Boundary Layer Theory, McGraw-Hill, New York, 1960.

[43]. B.E. Launder, D.B. Spalding, Mathematical models of turbulence, Academic Press, London and New York, 1972.

[44]. D.B. Spalding, A novel finite-difference formulation for differential expressions involving both first and second derivatives, Int. J. Num. Meth. Eng. 4 (1972), 551-559.

[45]. International Electrotechnical Commission, Industrial-process control valves - Flow capacity - Sizing Equations for Fluid under Installed Conditions, Report No. IEC 60534-2-1, 1998. 